\title{
Structure of the nucleon at leading and subleading twist in the covariant parton model
}

\author{
S. Bastami, ${ }^{1}$ A. V. Efremov, ${ }^{2}$ P. Schweitzer, ${ }^{1}$ O. V. Teryaev, ${ }^{2}$ and P. Zavada $\oplus^{3}$ \\ ${ }^{1}$ Department of Physics, University of Connecticut, Storrs, Connecticut 06269, USA \\ ${ }^{2}$ Bogoliubov Laboratory of Theoretical Physics, JINR, 141980 Dubna, Russia \\ ${ }^{3}$ Institute of Physics of the Czech Academy of Sciences, Na Slovance 2, 18221 Prague, Czech Republic
}

(Received 10 December 2020; accepted 18 December 2020; published 25 January 2021)

\begin{abstract}
The covariant parton model is generalized to describe quark correlators in a systematic way. Previous results are reproduced for the T-even leading-twist transverse momentum dependent parton distribution functions (TMDs), and for the first time all T-even twist-3 TMDs are evaluated in this model. We apply the approach to evaluate the fully unintegrated quark correlator which allows us to understand the modelspecific relations between different TMDs. We verify the consistency of the approach, present numerical results and compare to available TMD parametrizations.
\end{abstract}

DOI: 10.1103/PhysRevD.103.014024

\section{INTRODUCTION}

Transverse momentum dependent parton distribution functions (TMDs) allow one to explore the transverse structure of the nucleon in deeply inelastic scattering processes and are among the motivations for the planning of the Electron-Ion Collider [1]. They enter the description of processes like semi-inclusive deep-inelastic scattering (SIDIS) [2-4] (in conjunction with fragmentation functions [5]) or Drell-Yan [6,7] on the basis of TMD factorization theorems [8-22]. There has been an impressive progress in higher order QCD calculations [23-32] and phenomenological studies [33-46]. Aspects of TMD physics were reviewed in Refs. [47-52]. An important complement of the theoretical and phenomenological studies is provided by studies in models.

Models seek to explain the physics underlying phenomenological observations in simple terms by focusing on certain concepts. While often oversimplifying the complexities of hadronic physics, models are nevertheless insightful, guide our intuition, and deepen the understanding of nucleon structure. One value of the models is that they allow us to quantify how much of an observed phenomenon can be attributed to a specific model concept. Another important value is that models provide predictions for yet unknown nucleon properties which we can test in experiment.

Published by the American Physical Society under the terms of the Creative Commons Attribution 4.0 International license. Further distribution of this work must maintain attribution to the author(s) and the published article's title, journal citation, and DOI. Funded by SCOAP ${ }^{3}$.
This work deals with the study of TMDs in the covariant parton model (CPM) [53-65] originally developed as a model for the description of the hadronic tensor in DIS [5355]. It is a parton model in the sense that the partons are free and noninteracting. In the Feynman parton model this is the case in the infinite momentum frame [66]. In this sense the CPM goes one step further, and assumes the partons to be free in any frame. As free particles the partons are consequently on shell. At the heart of the CPM are two types of covariant functions, $G^{q}(P p)$ and $H^{q}(P p)$, which describe the distributions of the momenta of, respectively, unpolarized and polarized partons inside the nucleon. The covariant distributions are functions of the scalar $P p$ where $P$ is the nucleon and $p$ parton momentum.

The original formulation [53-55] allows one to evaluate the parton distribution functions (PDFs) $f_{1}^{a}(x), g_{1}^{a}(x), g_{T}^{a}(x)$ accessible through DIS structure functions (throughout this work we do not indicate the scale dependence explicitly). By an auxiliary polarized process due to the interference of vector and scalar currents, the approach was extended to the description of a hypothetical chiral-odd structure function and the transversity PDF $h_{1}^{a}(x)$ [56]. The model was further generalized by introducing the concept of "unintegrated structure functions," to describe twist-2 T-even TMDs $f_{1}^{a}\left(x, p_{T}\right), g_{1}^{a}\left(x, p_{T}\right), h_{1}^{a}\left(x, p_{T}\right), g_{1 T}^{\perp a}\left(x, p_{T}\right), h_{1 L}^{\perp a}\left(x, p_{T}\right)$, $h_{1 T}^{\perp a}\left(x, p_{T}\right)$ in [58]. Despite these generalizations, the limitation is that many TMDs especially at twist-3 level cannot be studied in this way because no (real or auxiliary) process is known how to compute them in a parton model framework like the CMP.

The purpose of this work (after a brief review of quark correlators and TMDs in Sec. II) is to generalize the formulation of the CPM to the description of quark correlators (in Sec. III), which will put us in the position 
to evaluate systematically all T-even TMDs. We will reproduce earlier model results for twist-2 TMDs, and derive new results for twist-3 TMDs (in Sec. IV). In order to test the internal theoretical consistency of the model we will investigate the various emerging relations among TMDs, some of which can be traced back to QCD equation-of-motion relations or the so-called Lorentzinvariance relations which must be valid in all quark models which respect Lorentz symmetry (in Sec. V). The relations among TMDs constitute one of the most interesting predictions of the CPM which can be tested quantitatively. We will make predictions for all T-even unpolarized and polarized twist-2 and twist-3 TMDs (in Sec. VI) and draw conclusions (in Sec. VII). Technical details are presented in the Appendix.

TMDs have been studied in bag [67-72], quark-diquark [73-81], chiral quark soliton [82-94], light-front constituent quark [95-102], Nambu-Jona-Lasinio [103], Valon [104], holographic [105,106], and quark-target [107111] models, and in some cases model independently in lattice QCD computations [112-119]. A parton model for PDFs that is similar to our study was discussed in Ref. [120]. We will compare to the results from other models.

In view of the variety of the approaches, it is interesting that some nonperturbative properties of TMDs are supported across a broad class of different models [121,122]. The results presented in this work contribute to a picture of TMDs emerging from models, and help to solidify the understanding of TMDs.

\section{QUARK CORRELATOR AND TMDS}

The quark correlation function is defined as

$$
\begin{aligned}
\Phi_{i j}^{q}(p, P, S)= & \int \frac{\mathrm{d}^{4} z}{(2 \pi)^{4}} \mathrm{e}^{i p z}\langle N(P, S)| \bar{\psi}_{j}^{q}(0) \\
& \times \mathcal{W}(0, z ; \text { path }) \psi_{i}^{q}(z)|N(P, S)\rangle,
\end{aligned}
$$

where $p$ is the quark momentum. $P$ and $S$ denote the nucleon momentum and polarization with $P^{2}=M^{2}, S^{2}=-1$, $P \cdot S=0$. The correlator depends furthermore on a lightlike four vector often denoted by $n^{\mu}$, which describes the light cone direction. The Wilson line $\mathcal{W}(0, z ;$ path $)$, which is symbolically indicated in (1), depends on $n^{\mu}$ and the considered process. Depending on the chosen path the correlator may be relevant for DIS, Drell-Yan, or another process [123-127]. Strictly speaking we should denote the correlator as $\Phi_{i j}^{q}(p, P, S, n)$, but for brevity we do not indicate the $n^{\mu}$ dependence (which is absent in the applications to quark models we have in mind).

Using light cone coordinates, $p^{ \pm}=\frac{1}{\sqrt{2}}\left(p^{0} \pm p^{1}\right)$, one introduces the integrated correlator

$\phi_{i j}^{q}\left(x, p_{T}, S\right)=\iint \mathrm{d} p^{-} \mathrm{d} p^{+} \Phi_{i j}^{q}(p, P, S) \delta\left(p^{+}-x P^{+}\right)$.

If we define $\phi^{q[\Gamma]}=\frac{1}{2} \operatorname{Tr}\left[\phi^{q}\left(x, p_{T}, S\right) \Gamma\right]$ then the leading twist quark TMDs are projected out from (2) as follows

$$
\begin{aligned}
\phi^{q\left[\gamma^{+}\right]} & =f_{1}^{q}-\frac{\varepsilon^{j k} p_{T}^{j} S_{T}^{k}}{M} f_{1 T}^{\perp q}, \\
\phi^{q\left[\gamma^{+} \gamma_{5}\right]} & =S_{L} g_{1}^{q}+\frac{\vec{p}_{T} \vec{S}_{T}}{M} g_{1 T}^{\perp q}, \\
\phi^{q\left[\sigma^{j+} \gamma_{5}\right]} & =S_{T}^{j} h_{1}^{q}+S_{L} \frac{p_{T}^{j}}{M} h_{1 L}^{\perp q}+\frac{\kappa^{j k} S_{T}^{k}}{M^{2}} h_{1 T}^{\perp q}+\frac{\varepsilon^{j k} p_{T}^{k}}{M} h_{1}^{\perp q},
\end{aligned}
$$

and the twist-3 quark TMDs are given by

$$
\begin{aligned}
\phi^{q[1]} & =\frac{M}{P^{+}}\left[e^{q}-\frac{\varepsilon^{j k} p_{T}^{j} S_{T}^{k}}{M} e_{T}^{\perp q}\right], \\
\phi^{q\left[i \gamma_{5}\right]} & =\frac{M}{P^{+}}\left[S_{L} e_{L}^{q}+\frac{\vec{p}_{T} \cdot \vec{S}_{T}}{M} e_{T}^{q}\right], \\
\phi^{a\left[\gamma^{j}\right]} & =\frac{M}{P^{+}}\left[\frac{p_{T}^{j}}{M} f^{\perp q}+\varepsilon^{j k} S_{T}^{k} f_{T}^{q}+S_{L} \frac{\varepsilon^{j k} p_{T}^{k}}{M} f_{L}^{\perp q}-\frac{\kappa^{j k} \varepsilon^{k l} S_{T}^{l}}{M^{2}} f_{T}^{\perp q}\right], \\
\phi^{q\left[\gamma^{j} \gamma_{5}\right]} & =\frac{M}{P^{+}}\left[S_{T}^{j} g_{T}^{q}+S_{L} \frac{p_{T}^{j}}{M} g_{L}^{\perp q}+\frac{\kappa^{j k} S_{T}^{k}}{M^{2}} g_{T}^{\perp q}+\frac{\varepsilon^{j k} p_{T}^{k}}{M} g^{\perp q}\right],
\end{aligned}
$$




$$
\begin{aligned}
\phi^{q\left[i \sigma^{j k} \gamma_{5}\right]} & =\frac{M}{P^{+}}\left[\frac{S_{T}^{j} p_{T}^{k}-S_{T}^{k} p_{T}^{j}}{M} h_{T}^{\perp q}-\varepsilon^{j k} h^{q}\right], \\
\phi^{q\left[i \sigma^{+-} \gamma_{5}\right]} & =\frac{M}{P^{+}}\left[S_{L} h_{L}^{q}+\frac{\vec{p}_{T} \cdot \vec{S}_{T}}{M} h_{T}^{q}\right] .
\end{aligned}
$$

The indices $j, k$ denote spatial directions transverse to the light cone, $\kappa^{j k}=\left(p_{T}^{j} p_{T}^{k}-\frac{1}{2} \delta^{j k} p_{T}^{2}\right)$ where $p_{T}^{2}=\left|\vec{p}_{T}\right|^{2}$, and $\varepsilon^{23}=-\varepsilon^{32}=1$ and zero else. In (3) it is understood that $f_{1}^{q}=f_{1}^{q}\left(x, p_{T}\right)$, etc. The T-even TMDs $f_{1}^{q}, g_{1}^{q}, g_{1 T}^{\perp q}, h_{1}^{q}, h_{1 L}^{\perp q}, h_{1 T}^{\perp q}$, $e^{q}, f^{\perp q}, g_{T}^{q}, g_{L}^{\perp q}, g_{T}^{\perp q}, h_{L}^{q}, h_{T}^{\perp q}, h_{T}^{q}$ can be computed in models based on quark degrees of freedom only. The other TMDs are T-odd, require explicit gauge field degrees of freedom, and cannot be modeled in the approach used in this work.

The fully unintegrated quark correlator (1) has the following expansion in terms of Lorentz-invariant amplitudes [128]

$$
\begin{aligned}
& \Phi^{q}(P, p, S)=M A_{1}^{q}+\not P A_{2}^{q}+\not p A_{3}^{q}+\frac{l}{2 M}[\not P, \not p] A_{4}^{q}+i(p \cdot S) \gamma_{5} A_{5}^{q}+M \not \gamma_{5} A_{6}^{q}
\end{aligned}
$$

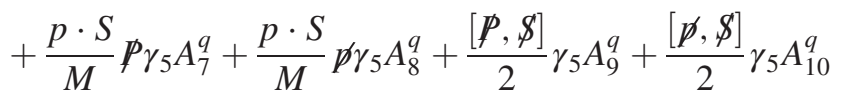

$$
\begin{aligned}
& +\frac{p \cdot S}{2 M^{2}}[\not P, \not p] \gamma_{5} A_{11}^{q}+\frac{1}{M} \varepsilon^{\mu \nu \rho \sigma} \gamma_{\mu} P_{\nu} p_{\rho} S_{\sigma} A_{12}^{q}+\mathcal{O}\left(B_{i}\right)
\end{aligned}
$$

where $\varepsilon^{0123}=1$. The amplitudes $A_{4}^{q}, A_{5}^{q}, A_{12}^{q}$ are T-odd. $\mathcal{O}\left(B_{i}\right)$ indicates symbolically the $B_{1}^{q}, \ldots, B_{20}^{q}$ amplitudes associated with the lightlike vector $n^{\mu}$ inherent in the Wilson line. In models without gauge field degrees of freedom T-odd $A_{i}$ and all $B_{i}$ amplitudes are absent, and T-even TMDs are expressed in terms of the T-even $A_{i}^{q}$ as

$$
\begin{aligned}
f_{1}^{q}\left(x, p_{T}\right) & =2 P^{+} \int \mathrm{d} p^{-}\left(A_{2}^{q}+x A_{3}^{q}\right), \\
g_{1}^{q}\left(x, p_{T}\right) & =2 P^{+} \int \mathrm{d} p^{-}\left(-A_{6}^{q}-\frac{P \cdot p-M^{2} x}{M^{2}}\left(A_{7}^{q}+x A_{8}^{q}\right)\right)+\mathcal{O}\left(B_{i}\right), \\
g_{1 T}^{\perp q}\left(x, p_{T}\right) & =2 P^{+} \int \mathrm{d} p^{-}\left(A_{7}+x A_{8}\right), \\
h_{1}^{q}\left(x, p_{T}\right) & =2 P^{+} \int \mathrm{d} p^{-}\left(-A_{9}^{q}-x A_{10}^{q}+\frac{\vec{p}_{T}^{2}}{2 M^{2}} A_{11}^{q}\right), \\
h_{1 L}^{\perp q}\left(x, p_{T}\right) & =2 P^{+} \int \mathrm{d} p^{-}\left(A_{10}^{q}-\frac{P \cdot p-M^{2} x}{M^{2}} A_{11}^{q}\right)+\mathcal{O}\left(B_{i}\right), \\
h_{1 T}^{\perp q}\left(x, p_{T}\right) & =2 P^{+} \int \mathrm{d} p^{-} A_{11}^{q}, \\
g_{L}^{\perp q}\left(x, p_{T}\right) & =2 P^{+} \int \mathrm{d} p^{-}\left(-\frac{P \cdot p-M^{2} x}{M^{2}} A_{8}^{q}\right)+\mathcal{O}\left(B_{i}\right), \\
e^{q}\left(x, p_{T}\right) & =2 P^{+} \int \mathrm{d} p^{-} A_{1}^{q}, \\
f^{\perp q}\left(x, p_{T}\right) & =2 P^{+} \int \mathrm{d} p^{-} A_{3}^{q}, \\
& =2 P^{+} \int \mathrm{d} p^{-}\left(-A_{6}^{q}+\frac{\vec{p}_{T}^{2}}{2 M^{2}} A_{8}^{q}\right), \\
& \\
& \\
&
\end{aligned}
$$




$$
\begin{aligned}
g_{T}^{\perp q}\left(x, p_{T}\right) & =2 P^{+} \int \mathrm{d} p^{-} A_{8}^{q}, \\
h_{T}^{\perp q}\left(x, p_{T}\right) & =2 P^{+} \int \mathrm{d} p^{-}\left(-A_{10}^{q}\right), \\
h_{L}^{q}\left(x, p_{T}\right) & =2 P^{+} \int \mathrm{d} p^{-}\left(-A_{9}^{q}-\frac{P \cdot p}{M^{2}} A_{10}^{q}+\left(\frac{P \cdot p-M^{2} x}{M^{2}}\right)^{2} A_{11}^{q}\right)+\mathcal{O}\left(B_{i}\right), \\
h_{T}^{q}\left(x, p_{T}\right) & =2 P^{+} \int \mathrm{d} p^{-}\left(-\frac{P \cdot p-M^{2} x}{M^{2}} A_{11}^{q}\right)+\mathcal{O}\left(B_{i}\right) .
\end{aligned}
$$

We only symbolically indicate the $B_{i}^{q}$ amplitudes as they are absent in quark models, cf. Ref. [129] for the full expressions. We also do not show the expressions for T-odd TMDs as they vanish in quark models with no explicit gluon degrees of freedom [130].

\section{FORMULATION OF THE COVARIANT PARTON MODEL}

We define the quark correlator in the CPM as follows

$$
\begin{aligned}
\Phi^{q}(p, P, S)_{i j}= & 2 P^{0} \Theta\left(p^{0}\right) \delta\left(p^{2}-m^{2}\right) \bar{u}_{j}(p) u_{i}(p) \\
& \times \begin{cases}\mathcal{G}^{q}(p P) & \text { unpolarized partons, } \\
\mathcal{H}^{q}(p P) & \text { polarized partons. }\end{cases}
\end{aligned}
$$

The prefactor $2 P^{0}$ is due to the covariant normalization $\left\langle N\left(P^{\prime}, S\right) \mid N(P, S)\right\rangle=2 P^{0}(2 \pi)^{3} \delta^{(3)}\left(\vec{P}^{\prime}-\vec{P}\right)$ of the nucleon states in Eq. (1). The onshell condition of the quarks in the CPM is implemented in terms of the Lorentz-invariant function $\Theta\left(p^{0}\right) \delta\left(p^{2}-m^{2}\right) . \mathcal{G}^{q}(p P)$ describes the covariant momentum distribution of unpolarized quarks of flavor $q=$ $u, d, \ldots$ inside the nucleon, while $\mathcal{H}^{q}(p P)$ describes the covariant distribution of polarized quarks.

In the CPM the quarks are on shell, which allows us to evaluate the bispinor expressions as

$$
\bar{u}(p) \Gamma u(p)=\operatorname{Tr}\left[\frac{1}{2}(\not p+m)\left(1+\gamma_{5} \not b\right) \Gamma\right] .
$$

xHere $\omega^{\mu}$ is the quark polarization vector which satisfies $\omega^{2}=-1$ and $p \cdot \omega=0$ and can be expressed in the CPM in terms of $p^{\mu}, P^{\mu}, S^{\mu}$ as follows [54]

$$
\omega^{\mu}=-\frac{M}{m} \frac{p \cdot S}{p \cdot P+m M} p^{\mu}-\frac{p \cdot S}{p \cdot P+m M} P^{\mu}+S^{\mu} .
$$

A more general expression for $\omega^{\mu}$ was given in [62] which coincides with (8) for massless quarks. In this work we will use Eq. (8) for $\omega^{\mu}$ and explore the more general representation for $\omega$ from [62] elsewhere.

By exploring Eq. (7) we obtain the following compact expression

$$
\begin{aligned}
\operatorname{Tr}\left[\Phi^{q}(p, P, S) \Gamma\right] & =P^{0} \Theta\left(p^{0}\right) \delta\left(p^{2}-m^{2}\right) \operatorname{Tr}\left[( \not p + m ) \left(\mathcal{G}^{q}(p P)\right.\right. \\
& \left.\left.+\mathcal{H}^{q}(p P) \gamma_{5} \phi\right) \Gamma\right]
\end{aligned}
$$

We recall that the covariant function $\mathcal{G}^{q}(p P)$ is positive and has a partonic interpretation within the CPM: when interpreted in the nucleon rest frame it describes the momentum distribution of unpolarized quarks in the nucleon. Similarly $\mathcal{H}^{q}(p P)$ describes the momentum distribution of polarized $q$ in a nucleon polarized in its rest frame along $S^{\mu}=(0, \vec{S})$ [53-55]. The unpolarized covariant function satisfies $G^{q}(P p) \geq 0$, and the polarized one $\left|H^{q}(P p)\right| \leq G^{q}(P p)$ which reflects the partonic interpretation.

Equations (6)-(9) can be viewed as a definition of the CPM and describe how to evaluate in the model quark correlation functions. In the remainder of this work, we will compute all twist-2 and twist-3 T-even TMDs of quarks on the basis of Eqs. (6)-(9). Hereby we will reproduce results known from previous works, derive many new results (especially for twist-3 TMDs), and demonstrate the internal theoretical consistency of the approach.

\section{QUARK TMDS IN THE COVARIANT PARTON MODEL}

This section is devoted to quark TMDs. In the twist-2 case we will rederive results obtained in Ref. [58] in different ways. In the twist-3 case we will (with one exception) present new predictions.

\section{A. The unpolarized leading twist TMD $f_{1}^{q}\left(x, p_{T}\right)$}

In order to derive the expression for the TMD $f_{1}^{q}\left(x, p_{T}\right)$ we evaluate the correlator 


$$
\begin{aligned}
\phi^{q\left[\gamma^{+}\right]}\left(x, \vec{p}_{T}, S\right) & =\int \mathrm{d} p^{-} \mathrm{d} p^{+} P^{0} \mathcal{G}^{q}(p P) \Theta\left(p^{0}\right) \delta\left(p^{2}-m^{2}\right) \delta\left(p^{+}-x P^{+}\right) \bar{u}(p) \gamma^{+} u(p), \\
& =\int \mathrm{d} p^{-} \mathrm{d} p^{+} \frac{P^{0} \mathcal{G}^{q}(p P)}{P^{+}} \Theta\left(p^{0}\right) \delta\left(p^{2}-m^{2}\right) \delta\left(x-\frac{p^{+}}{P^{+}}\right) \bar{u}(p) \gamma^{+} u(p) .
\end{aligned}
$$

We choose to work in the nucleon rest frame where $p P=p^{0} M$. In the following we will denote the covariant function $\mathcal{G}^{q}(p P)$ in the nucleon rest frame for simplicity by $\mathcal{G}^{q}\left(p^{0}\right)$. In the nucleon rest frame $p^{+} / P^{+}=\left(p^{0}+p^{1}\right) / M$. To arrive at the formulation of the CPM from prior works we change the integration variables $\mathrm{d} p^{-} \mathrm{d} p^{+} \rightarrow \mathrm{d} p^{0} \mathrm{~d} p^{1}$ such that

$$
\iint \mathrm{d} p^{-} \mathrm{d} p^{+} \Theta\left(p^{0}\right) \delta\left(p^{2}-m^{2}\right) \ldots=\iint \mathrm{d} p^{0} \mathrm{~d} p^{1} \Theta\left(p^{0}\right) \delta\left(\left(p^{0}\right)^{2}-\vec{p}^{2}-m^{2}\right) \ldots=\left.\int \frac{\mathrm{d} p^{1}}{2 p^{0}} \ldots\right|_{p^{0}=\sqrt{\vec{p}^{2}+m^{2}}} .
$$

Evaluating the bispinor expression $\bar{u}(p) \gamma^{\mu} u(p)=2 p^{\mu}$ we obtain

$$
\phi^{q\left[\gamma^{+}\right]}\left(x, \vec{p}_{T}, S\right)=f_{1}^{q}\left(x, p_{T}\right)=\int \frac{\mathrm{d} p^{1}}{p^{0}} \mathcal{G}^{q}\left(p^{0}\right) \delta\left(x-\frac{p^{0}+p^{1}}{M}\right)\left(p^{0}+p^{1}\right) .
$$

This coincides with the result for $f_{1}^{q}\left(x, p_{T}\right)$ from Eq. (25) in [58] (after the substitution $p^{1} \rightarrow\left(-p^{1}\right)$ ). In [58] this result was obtained by introducing and modeling a " $p_{T}$-unintegrated" hadronic tensor. Here we derive the same result systematically from the model expression of the quark correlator (9). We also see that in the model $\phi^{q\left[\gamma^{+}\right]}\left(x, \vec{p}_{T}, S\right)$ has no term proportional to $\vec{p}_{T} \vec{S}_{T}$ and hence the Sivers function is zero as expected in models with no gluons [130].

\section{B. The chiral-even polarized leading twist TMDs $g_{1}^{q}\left(x, p_{T}\right)$ and $g_{1 T}^{\perp q}\left(x, p_{T}\right)$}

These TMDs require polarization. We use the expression (8) for the quark polarization vector $\omega^{\mu}$ in nucleon rest frame where $^{1} S^{\mu}=\left(0, S_{L}, \vec{S}_{T}\right)$ in usual four-vector notation. We explore (11) and $\bar{u}(p) \gamma^{\mu} \gamma_{5} u(p)=2 \omega^{\mu}$, and obtain

$$
\begin{aligned}
\phi^{q\left[\gamma^{+} \gamma_{5}\right]}\left(x, \vec{p}_{T}, S\right)= & S_{L} \int \frac{\mathrm{d} p^{1}}{p^{0}} \mathcal{H}^{q}\left(p^{0}\right) \delta\left(x-\frac{p^{0}+p^{1}}{M}\right)\left[\frac{p^{1}\left(p^{0}+p^{1}\right)}{p^{0}+m}+\frac{p^{1} m}{p^{0}+m}+m\right] \\
& +\vec{p}_{T} \vec{S}_{T} \int \frac{\mathrm{d} p^{1}}{p^{0}} \mathcal{H}^{q}\left(p^{0}\right) \delta\left(x-\frac{p^{0}+p^{1}}{M}\right)\left[\frac{p^{0}+p^{1}}{p^{0}+m}+\frac{m}{p^{0}+m}\right],
\end{aligned}
$$

where we grouped terms proportional to longitudinal and transverse polarization. Comparing to the coefficients in Eq. (3b) we read off the model results for $g_{1}^{q}\left(x, p_{T}\right)$ and $g_{1 T}^{\perp q}\left(x, p_{T}\right)$ which, after some algebra, can be written as

$$
\begin{aligned}
g_{1}^{q}\left(x, p_{T}\right) & =\int \frac{\mathrm{d} p^{1}}{p^{0}} \mathcal{H}^{q}\left(p^{0}\right) \delta\left(x-\frac{p^{0}+p^{1}}{M}\right)\left[p^{0}+p^{1}-\frac{p_{T}^{2}}{p^{0}+m}\right], \\
g_{1 T}^{\perp q}\left(x, p_{T}\right) & =\int \frac{\mathrm{d} p^{1}}{p^{0}} \mathcal{H}^{q}\left(p^{0}\right) \delta\left(x-\frac{p^{0}+p^{1}}{M}\right)\left[M \frac{p^{0}+p^{1}+m}{p^{0}+m}\right],
\end{aligned}
$$

and coincide with the expressions in Eqs. (16), (17) of Ref. [58]. It is important to stress that these results were obtained from the antisymmetric part of the " $p_{T}$-unintegrated" hadronic tensor, which was constructed and modeled in [58], while here they follow straightforwardly from the model expression of the quark correlator (9).

\section{The chiral-odd polarized leading twist TMDs $h_{1}^{q}\left(x, p_{T}\right), h_{1 L}^{\perp q}\left(x, p_{T}\right), h_{1 T}^{\perp q}\left(x, p_{T}\right)$}

In order to evaluate the correlator $\phi^{\left[i \sigma^{+j}\right]}$ we proceed as in (11), explore $\bar{u}(p) i \sigma^{\mu \nu} u(p)=2\left(\omega^{\mu} p^{\nu}-\omega^{\nu} p^{\mu}\right)$, and insert the expression (8) for $\omega^{\mu}$ with $S^{\mu}=\left(0, S_{L}, \vec{S}_{T}\right)$. This yields

\footnotetext{
${ }^{1}$ In Ref. [58] the light cone spatial direction was chosen opposite to our work; i.e., the signs of the first components of all vectors are reversed: for instance $\left.\left(p^{0}-p^{1}\right)\right|_{\text {Ref. [58] }}$ corresponds to $\left.\left(p^{0}+p^{1}\right)\right|_{\text {here }}$. Consequently the conventions are such that $\left.S_{L}\right|_{\text {here }}=-\left.S_{L}\right|_{\text {Ref. [58] }}$.
} 


$$
\begin{aligned}
\phi^{q\left[\sigma^{+j}\right]}\left(x, \vec{p}_{T}, S\right) & =\int \frac{\mathrm{d} p^{1}}{p^{0}} \mathcal{H}^{q}\left(p^{0}\right) \delta\left(x-\frac{p^{0}+p^{1}}{M}\right)\left(\left(\omega^{0}+\omega^{1}\right) p^{j}-\omega^{i}\left(p^{0}+p^{1}\right)\right) \\
& =\int \frac{\mathrm{d} p^{1}}{p^{0}} \mathcal{H}^{q}\left(p^{0}\right) \delta\left(x-\frac{p^{0}+p^{1}}{M}\right)\left(S_{T}^{j}\left(p^{0}+p^{1}\right)-\frac{\left(p^{0}+p^{1}+m\right)}{\left(p^{0}+m\right)} S_{L} p_{T}^{j}-\frac{\vec{p}_{T} \vec{S}_{T}}{\left(p^{0}+m\right)} p_{T}^{j}\right)
\end{aligned}
$$

Notice that $\left(\vec{p}_{T} \cdot \vec{S}_{T}\right) p_{T}^{j}=\frac{1}{2} \vec{p}_{T}^{2} S_{T}^{j}+\left(p_{T}^{j} p_{T}^{k}-\frac{1}{2} \delta^{j k} \vec{p}_{T}^{2}\right) S_{T}^{k}$ where the first term (monopole in $\vec{p}_{T}$ ) contributes to transversity while the second term (quadrupole structure) gives rise to pretzelosity. Comparing to the correlator (3c), we read off the results

$$
\begin{aligned}
h_{1}^{q}\left(x, p_{T}\right) & =\int \frac{\mathrm{d} p^{1}}{p^{0}} \mathcal{H}^{q}\left(p^{0}\right) \delta\left(x-\frac{p^{0}+p^{1}}{M}\right)\left(p^{0}+p^{1}-\frac{p_{T}^{2}}{2\left(p^{0}+m\right)}\right), \\
h_{1 L}^{\perp q}\left(x, p_{T}\right) & =\int \frac{\mathrm{d} p^{1}}{p^{0}} \mathcal{H}^{q}\left(p^{0}\right) \delta\left(x-\frac{p^{0}+p^{1}}{M}\right)\left(-M \frac{p^{0}+p^{1}+m}{p^{0}+m}\right), \\
h_{1 T}^{\perp q}\left(x, p_{T}\right) & =\int \frac{\mathrm{d} p^{1}}{p^{0}} \mathcal{H}^{q}\left(p^{0}\right) \delta\left(x-\frac{p^{0}+p^{1}}{M}\right)\left(-\frac{M^{2}}{p^{0}+m}\right) .
\end{aligned}
$$

As the model generates no unpolarized structure in the correlator (3c), the T-odd Boer-Mulders function $h_{1}^{\perp q}\left(x, p_{T}\right)$ vanishes as expected in quark models [130]. The results (16) agree with those obtained previously by generalizing the auxiliary polarized process due to interference of vector and scalar currents to the " $p_{T}$-unintegrated" situation [58]. Notice that the results can be simplified using, e.g., $p^{0}+p^{1}=x M$ under the integrals in (16).

\section{Twist-3 TMDs}

In the twist- 3 correlators $(3 \mathrm{~d})-(3 \mathrm{~g})$ we encounter two new Dirac structures, $\bar{u}(p) \rrbracket u(p)=2 m$ related to $e^{q}\left(x, p_{T}\right)$ and $\bar{u}(p) i \gamma_{5} u(p)=0$ for an on shell particle. Proceeding analog to Secs. IVA-IV C yields the following results

$$
\begin{aligned}
f^{\perp q}\left(x, p_{T}\right) & =\int \frac{\mathrm{d} p^{1}}{p^{0}} \mathcal{H}^{q}\left(p^{0}\right) \delta\left(x-\frac{p^{0}+p^{1}}{M}\right) \frac{M\left(p^{0}+m\right)}{p^{0}+m}, \\
e^{q}\left(x, p_{T}\right) & =\int \frac{\mathrm{d} p^{1}}{p^{0}} \mathcal{H}^{q}\left(p^{0}\right) \delta\left(x-\frac{p^{0}+p^{1}}{M}\right) \frac{m\left(p^{0}+m\right)}{p^{0}+m}, \\
g_{T}^{q}\left(x, p_{T}\right) & =\int \frac{\mathrm{d} p^{1}}{p^{0}} \mathcal{H}^{q}\left(p^{0}\right) \delta\left(x-\frac{p^{0}+p^{1}}{M}\right) \frac{m\left(p^{0}+m\right)+\frac{1}{2} \vec{p}_{T}^{2}}{p^{0}+m}, \\
g_{T}^{\perp q}\left(x, p_{T}\right) & =\int \frac{\mathrm{d} p^{1}}{p^{0}} \mathcal{H}^{q}\left(p^{0}\right) \delta\left(x-\frac{p^{0}+p^{1}}{M}\right) \frac{M^{2}}{p^{0}+m}, \\
g_{L}^{\perp q}\left(x, p_{T}\right) & =\int \frac{\mathrm{d} p^{1}}{p^{0}} \mathcal{H}^{q}\left(p^{0}\right) \delta\left(x-\frac{p^{0}+p^{1}}{M}\right) \frac{M\left(M x-p^{0}\right)}{p^{0}+m}, \\
h_{T}^{\perp q}\left(x, p_{T}\right) & =\int \frac{\mathrm{d} p^{1}}{p^{0}} \mathcal{H}^{q}\left(p^{0}\right) \delta\left(x-\frac{p^{0}+p^{1}}{M}\right) \frac{M\left(p^{0}+m\right)}{p^{0}+m}, \\
h_{L}^{q}\left(x, p_{T}\right) & =\int \frac{\mathrm{d} p^{1}}{p^{0}} \mathcal{H}^{q}\left(p^{0}\right) \delta\left(x-\frac{p^{0}+p^{1}}{M}\right) \frac{m\left(p^{0}+m\right)+\vec{p}_{T}^{2}}{p^{0}+m}, \\
h_{T}^{q}\left(x, p_{T}\right) & =\int \frac{\mathrm{d} p^{1}}{p^{0}} \mathcal{H}^{q}\left(p^{0}\right) \delta\left(x-\frac{p^{0}+p^{1}}{M}\right) \frac{M\left(p^{0}-M x\right)}{p^{0}+m} .
\end{aligned}
$$


Only the model expression for the twist-3 TMD $g_{T}^{q}\left(x, p_{T}\right)$ was computed before in the CPM, as it is related to the ("unintegrated" generalization of the) hadronic tensor in polarized DIS, and our result (17c) agrees with the expression from [58]. The results for the other twist-3 TMDs are new. Notice that also in twist- 3 case T-odd TMDs vanish in the CPM as it must be for a model with no explicit gauge field degrees of freedom [130].

\section{RELATIONS AMONG TMDS}

In QCD the different TMDs are all independent of each other, and describe different aspects of the nucleon structure. Due to the simpler dynamics or additional symmetries, different TMDs can be related to each other in quark models. The goal of this section is to discuss the relations among TMDs in the CPM.

\section{A. Equation-of-motion relations}

An important consistency test of the model results is provided by the following relations which can be derived by making use of the QCD equations of motion (EOM) and are given by

$$
\begin{aligned}
x e^{q}\left(x, p_{T}\right) & =x \tilde{e}^{q}\left(x, p_{T}\right)+\frac{m}{M} f_{1}^{q}\left(x, p_{T}\right), \\
x f^{\perp q}\left(x, p_{T}\right) & =x \tilde{f}^{\perp q}\left(x, p_{T}\right)+f_{1}^{q}\left(x, p_{T}\right), \\
x g_{L}^{\perp q}\left(x, p_{T}\right) & =x \tilde{g}_{L}^{\perp q}\left(x, p_{T}\right)+g_{1}^{q}\left(x, p_{T}\right)+\frac{m}{M} h_{1 L}^{\perp q}\left(x, p_{T}\right),
\end{aligned}
$$

$$
x g_{T}^{q}\left(x, p_{T}\right)=\tilde{g}_{T}^{q}\left(x, p_{T}\right)+g_{1 T}^{\perp(1) q}\left(x, p_{T}\right)+\frac{m}{M} h_{1}^{q}\left(x, p_{T}\right),
$$

$$
x g_{T}^{\perp q}\left(x, p_{T}\right)=x \tilde{g}_{T}^{\perp q}\left(x, p_{T}\right)+g_{1 T}^{\perp q}\left(x, p_{T}\right)+\frac{m}{M} h_{1 T}^{\perp q}\left(x, p_{T}\right),
$$

$$
x h_{L}^{q}\left(x, p_{T}\right)=x \tilde{h}_{L}^{q}\left(x, p_{T}\right)-2 h_{1 L}^{\perp(1) q}\left(x, p_{T}\right)+\frac{m}{M} g_{1}^{q}\left(x, p_{T}\right),
$$

$$
\begin{aligned}
x h_{T}^{q}\left(x, p_{T}\right)= & x \tilde{h}_{T}^{q}\left(x, p_{T}\right)-h_{1}^{q}\left(x, p_{T}\right)-h_{1 T}^{\perp(1)}\left(x, p_{T}\right) \\
& +\frac{m}{M} g_{1 T}^{\perp}\left(x, p_{T}\right)
\end{aligned}
$$

$x h_{T}^{\perp q}\left(x, p_{T}\right)=x \tilde{h}_{T}^{\perp q}\left(x, p_{T}\right)+h_{1}^{q}\left(x, p_{T}\right)-h_{1 T}^{\perp(1)}\left(x, p_{T}\right)$,

where the transverse moment $n$ of a generic TMD $f^{q}\left(x, p_{T}\right)$ is defined as follows

$$
f^{(n) q}\left(x, p_{T}\right)=\left(\frac{p_{T}^{2}}{2 M^{2}}\right)^{n} f^{q}\left(x, p_{T}\right)
$$

The EOMs (18) arise because the quark correlators defining twist-3 TMDs, Eqs. (3d)-(3g), can be decomposed into contributions from quark-gluon correlators, twist-2 correlators, and terms proportional to current quark masses by exploring QCD equations of motion [4]. The quark-gluon correlators give rise to "genuine twist- 3 " contributions or "interaction dependent terms" which are denoted by tildefunctions in (18). In general the EOMs do not imply relations among TMDs, but define the respective genuine twist-3 tilde contributions.

In quark models in general the tilde terms are nonzero and arise from the model interactions due to the pertinent model equations of motion. The EOM of the CPM is the free Dirac equation, which implies the absence of tilde terms. Our results for twist-2 and twist-3 TMDs, Eqs. (12), (14), (16), (17), satisfy the EOM relations (18) with the tilde terms set to zero. This is an important consistency test for our new results for all twist-3 TMDs.

\section{B. Lorentz invariance relations}

In models with no gluonic degrees of freedom, such as the CPM, T-odd TMDs are absent [130] and the quark correlator can be decomposed in terms of nine T-even $A_{i}$ amplitudes while it gives rise to 14 T-even TMDs, see Sec. II. As there are 14 TMDs and 9 linearly independent amplitudes, this implies five relations among T-even TMDs. These relations are referred to as Lorentz-invariance relations (LIRs) and are not valid in QCD [131] but must hold in all models that preserve Lorentz symmetry and exhibit no gauge degrees of freedom. The LIRs are given by [3]

$$
\begin{gathered}
g_{T}^{q}(x) \stackrel{\text { LIR }}{=} g_{1}^{q}(x)+\frac{\mathrm{d}}{\mathrm{d} x} g_{1 T}^{\perp(1) q}(x), \\
h_{L}^{q}(x) \stackrel{\mathrm{LIR}}{=} h_{1}^{q}(x)-\frac{\mathrm{d}}{\mathrm{d} x} h_{1 L}^{\perp(1) q}(x), \\
h_{T}^{q}(x) \stackrel{\mathrm{LIR}}{=}-\frac{\mathrm{d}}{\mathrm{d} x} h_{1 T}^{\perp(1) q}(x), \\
g_{L}^{\perp q}(x)+\frac{\mathrm{d}}{\mathrm{d} x} g_{T}^{\perp(1) q}(x) \stackrel{\text { LIR }}{=} 0, \\
h_{T}^{q}\left(x, p_{T}\right)-h_{T}^{\perp q}\left(x, p_{T} \stackrel{\stackrel{\mathrm{LIR}}{=}}{=} h_{1 L}^{\perp q}\left(x, p_{T}\right),\right.
\end{gathered}
$$

and connect twist-3 TMDs (on left-hand sides of the above equations) with twist-2 TMDs (if any, on right-hand sides). The CPM satisfies all LIRs. This is an important consistency test for the model. Equations (20a)-(20d) can be proven using the methods developed in Appendix $\mathrm{C}$ of 
Ref. [58], while (20e) corresponds to the quark model relation (21f) that is discussed below.

\section{Quark model relations}

Our results for the twist-2 and twist-3 TMDs satisfy also the following relations [72]

$$
\begin{gathered}
g_{1 T}^{\perp q}\left(x, p_{T}\right)=-h_{1 L}^{\perp q}\left(x, p_{T}\right), \\
g_{T}^{\perp q}\left(x, p_{T}\right)=-h_{1 T}^{\perp q}\left(x, p_{T}\right), \\
g_{L}^{\perp q}\left(x, p_{T}\right)=-h_{T}^{q}\left(x, p_{T}\right), \\
g_{1}^{q}\left(x, p_{T}\right)-h_{1}^{q}\left(x, p_{T}\right)=h_{1 T}^{\perp(1) q}\left(x, p_{T}\right), \\
g_{T}^{q}\left(x, p_{T}\right)-h_{L}^{q}\left(x, p_{T}\right)=h_{1 T}^{\perp(1) q}\left(x, p_{T}\right), \\
h_{T}^{q}\left(x, p_{T}\right)-h_{T}^{\perp q}\left(x, p_{T}\right)=h_{1 L}^{\perp q}\left(x, p_{T}\right) .
\end{gathered}
$$

These relations are valid in a large class of quark models, including spectator models, bag model, light-front constituent quark model $[60,72,73,95,121]$. The CPM also supports the following nonlinear quark model relations

$$
\begin{aligned}
h_{1}^{q}\left(x, p_{T}\right) h_{1 T}^{\perp q}\left(x, p_{T}\right)= & -\frac{1}{2}\left[h_{1 L}^{\perp q}\left(x, p_{T}\right)\right]^{2}, \\
g_{T}^{q}\left(x, p_{T}\right) g_{T}^{\perp q}\left(x, p_{T}\right)= & +\frac{1}{2}\left[g_{1 T}^{\perp q}\left(x, p_{T}\right)\right]^{2} \\
& -g_{1 T}^{\perp q}\left(x, p_{T}\right) g_{L}^{\perp q}\left(x, p_{T}\right) .
\end{aligned}
$$

In [58] it was shown that the CPM complies with the relations (21a), (21d), (21g) featuring only twist-2 TMDs. Here we see that the model supports the full set of linear and nonlinear quark model relations (21).

One can impose the additional assumption of the $\mathrm{SU}(4)$ spin-flavor symmetry in the CPM by assuming $\mathcal{G}^{q}(p P)=$ $N^{q} \mathcal{F}(p P)$ and $\mathcal{G}^{q}(p P)=P^{q} \mathcal{F}(p P)$. For proton the $\mathrm{SU}(4)$ spin flavor factors are given by [132]

$$
\begin{aligned}
N_{u}=\frac{N_{c}+1}{2}, & N_{d}=\frac{N_{c}-1}{2}, \\
P_{u}=\frac{N_{c}+5}{6}, & P_{d}=\frac{-N_{c}+1}{6},
\end{aligned}
$$

and those for neutron follow from interchanging $u \leftrightarrow d$. Under the assumption of SU(4) spin-flavor symmetry and introducing the definition $\mathcal{D}^{q}=P^{q} / N^{q}$ also the following $\mathrm{SU}(4)$ quark model relations equations hold in the CPM

$$
\begin{aligned}
\mathcal{D}^{q} f_{1}^{q}\left(x, p_{T}\right)+g_{1}^{q}\left(x, p_{T}\right) & =2 h_{1}^{q}\left(x, p_{T}\right), \\
\mathcal{D}^{q} e^{q}\left(x, p_{T}\right)+h_{L}^{q}\left(x, p_{T}\right) & =2 g_{T}^{q}\left(x, p_{T}\right), \\
\mathcal{D}^{q} f^{\perp q}\left(x, p_{T}\right) & =h_{T}^{\perp q}\left(x, p_{T}\right) .
\end{aligned}
$$

It is important to stress that relations connecting unpolarized and polarized TMDs such as (23) require the stronger additional assumption of SU(4) spin-flavor symmetry.

The deeper reason for the appearance of the quark model relations including twist-2 TMDs, (21a), (21d), (21d) [and (23a) under the additional assumption of SU(4) spin-flavor symmetry], can be traced back to the symmetries of the light cone wave functions in a large class of independentparticle models where the quarks do not interact with each other but are bound by a mean field [121]. Not all models support these relations. A counterexample are quark-target models [108] where the relations (21), (23) are not valid.

\section{Wandzura-Wilczek relations}

In the CPM we can derive the following WW relations (the abbreviation WW is explained below)

$g_{T}^{q}(x) \stackrel{\mathrm{WW}}{=} \int_{x}^{1} \frac{\mathrm{d} y}{y} g_{1}^{q}(y)+\frac{m}{M}\left[-\frac{h_{1}^{q}(x)}{x}+\int_{x}^{1} \frac{\mathrm{d} y}{y^{2}} h_{1}^{q}(y)\right]$,

$h_{L}^{q}(x) \stackrel{\mathrm{WW}}{=} 2 x \int_{x}^{1} \frac{\mathrm{d} y}{y^{2}} h_{1}^{q}(y)+\frac{m}{M}\left[\frac{g_{1}^{q}(x)}{x}-2 x \int_{x}^{1} \frac{\mathrm{d} y}{y^{3}} g_{1}^{q}(y)\right]$.

Equation (24a) is obtained by integrating (18d) over $p_{T}$ with $\tilde{g}_{T}^{q}(x)=0$, solving for $g_{1 T}^{\perp(1) q}(x)$ and inserting the result into (20a) and finally integrating $\frac{\mathrm{d}}{\mathrm{d} x} g_{T}^{q}(x)$. Similarly Eq. (24b) is derived from the EOM relation (18f) and the LIR (20b). As they follow from EOM relations and LIRs, the WW relations (24) contain no new information.

From the point of view of QCD the relations (24) are of interest, as they constitute the so-called Wandzura-Wilczek (WW) approximation in QCD based on the neglect of tilde terms (and in practice also mass terms) [67,133]. This approximation is supported by instanton vacuum calculations $[134,135]$ where the tilde terms $\tilde{g}_{T}^{q}(x)$ and $\tilde{h}_{L}^{q}(x)$ are small compared to the twist-2 terms in (24), see also Ref. [136]. The smallness of $\tilde{g}_{T}^{q}(x)$ is supported by lattice QCD studies [137,138], and experiment [139-141] which indicate that $\tilde{g}_{T}^{q}(x)$ and $\tilde{h}_{L}^{q}(x)$ are small compared to the twist- 2 terms in (24). In the CPM the tilde terms are exactly zero, and the relations (24) hold exactly. Further discussions of WW relations in various contexts can be found in Refs. [142-150].

For completeness we remark that the assumption that tilde terms (and mass terms) are small and numerically negligible has been also applied to TMDs [129,151-157]. In this case one speaks of Wandzura-Wilczek-type approximations. Neglecting current quark mass effects, two examples of such relations are [154]

$$
g_{1 T}^{\perp(1) q}(x) \stackrel{\text { WW-type }}{=} x \int_{x}^{1} \frac{\mathrm{d} y}{y} g_{1}^{q}(y),
$$




$$
h_{1 L}^{\perp(1) q}(x) \stackrel{\text { WW-type }}{=}-2 x^{2} \int_{x}^{1} \frac{\mathrm{d} y}{y^{2}} h_{1}^{q}(y) .
$$

Such approximations can be tested in SIDIS and Drell-Yan experiments [156-158]. In the CPM the relations (25) are exact.

\section{E. Independent amplitudes in the covariant parton model}

The previous sections have shown that the CPM supports many relations. It is an interesting question how many independent TMDs exist in this model. For that we evaluate the amplitudes of the unintegrated correlator (4). As expected, the T-odd $A_{4}^{q}, A_{5}^{q}, A_{12}^{q}$ are zero. For the T-even amplitudes we obtain

$$
\begin{aligned}
& A_{1}^{q}=P^{0} \Theta\left(p^{0}\right) \delta\left(p^{2}-m^{2}\right) \mathcal{G}^{q}(p P) \frac{m}{M} \\
& A_{2}^{q}=0 \\
& A_{3}^{q}=P^{0} \Theta\left(p^{0}\right) \delta\left(p^{2}-m^{2}\right) \mathcal{G}^{q}(p P) \\
& A_{6}^{q}=P^{0} \Theta\left(p^{0}\right) \delta\left(p^{2}-m^{2}\right) \mathcal{H}^{q}(p P)\left(-\frac{m}{M}\right) \\
& A_{7}^{q}=P^{0} \Theta\left(p^{0}\right) \delta\left(p^{2}-m^{2}\right) \mathcal{H}^{q}(p P) \frac{m M}{p \cdot P+m M} \\
& A_{8}^{q}=P^{0} \Theta\left(p^{0}\right) \delta\left(p^{2}-m^{2}\right) \mathcal{H}^{q}(p P) \frac{M^{2}}{p \cdot P+m M} \\
& A_{9}^{q}=0 \\
& A_{10}^{q}=P^{0} \Theta\left(p^{0}\right) \delta\left(p^{2}-m^{2}\right) \mathcal{H}^{q}(p P)(-1) \\
& A_{11}^{q}=P^{0} \Theta\left(p^{0}\right) \delta\left(p^{2}-m^{2}\right) \mathcal{H}^{q}(p P)\left(-\frac{M^{2}}{p \cdot P+m M}\right)
\end{aligned}
$$

Interestingly the T-even amplitudes $A_{2}^{q}, A_{9}^{q}$ also vanish, because our model does not generate these Lorentz structures. The remaining seven nonzero T-even amplitudes are related to each other by five relations. In the unpolarized case we have two nonzero amplitudes related by 1 relation,

$$
A_{1}^{q}=\frac{m}{M} A_{3}^{q} .
$$

In the polarized case we have five nonzero amplitudes related by four relations,

$$
\begin{aligned}
A_{6}^{q} & =-\frac{m}{M} A_{10}^{q}, \\
A_{7}^{q} & =+\frac{m}{M} A_{8}^{q}, \\
A_{8}^{q} & =-A_{11}^{q}, \\
A_{10}^{q} & =-\frac{p P+m M}{M^{2}} A_{8}^{q} .
\end{aligned}
$$

Notice that for an on shell particle the relation $P \cdot p=$ $\frac{\vec{p}_{T}^{2}+m^{2}}{2 x}+\frac{1}{2} x M^{2}$ holds. Thus in the model (but not in general) the product $P \cdot p$ does not depend on $p^{-}$and can be pulled out of the integrals over $p^{-}$in (5). Inserting the results (26) for the amplitudes into the expressions (5), we recover the results obtained in Eqs. (12), (14), (16), (17), which provide an independent test of the model.

\section{F. Independent TMDs in the model}

The previous section has shown that there are two independent amplitudes: one unpolarized and one polarized. Consequently, all unpolarized TMDs are related to each other, and all polarized TMDs are related to each other. Thus it is possible to choose one unpolarized and one polarized TMD, and express all other TMDs in terms of them.

In the unpolarized case we have three T-even TMDs $f_{1}^{q}\left(x, p_{T}\right), e^{q}\left(x, p_{T}\right), f^{\perp q}\left(x, p_{T}\right)$. We can choose the wellknown unpolarized twist-2 TMDs as basis function. The other unpolarized T-even TMDs are then given by

$$
\begin{gathered}
x e^{q}\left(x, p_{T}\right) \stackrel{\mathrm{CPM}}{=} \frac{m}{M} f_{1}^{q}\left(x, p_{T}\right), \\
x f^{\perp q}\left(x, p_{T}\right) \stackrel{\mathrm{CPM}}{=} f_{1}^{q}\left(x, p_{T}\right) .
\end{gathered}
$$

Notice that $e^{q}\left(x, p_{T}\right)$ vanishes if we one neglects current quark mass effects.

In the polarized T-even sector we have 11 TMDs. At first glance it would seem natural to express all polarized TMDs in terms of the relatively well-known helicity distribution $g_{1}^{q}\left(x, p_{T}\right)$. This is possible, but not ideal for the following reason. In the CPM, $g_{1}^{q}\left(x, p_{T}\right)$ exhibits a node at $p_{T}=x M$ (neglecting current quark masses) [61]. Consequently TMDs without nodes would be expressed in terms $g_{1}^{q}\left(x, p_{T}\right)$ divided by a prefactor which is singular at $p_{T}=$ $x M$ in order to remove the node present in $g_{1}^{q}\left(x, p_{T}\right)$. This is impractical for phenomenological applications.

It is clearly an advantage to choose a TMD without a node to express the other TMDs. A convenient choice for a basis function for polarized TMDs is transversity $h_{1}^{q}\left(x, p_{T}\right)$ which exhibits no node in the model and is, after $g_{1}^{q}\left(x, p_{T}\right)$, the currently best known T-even TMD. It is convenient to neglect current quark mass effects, which make the explicit expressions quite bulky and can be safely expected to be small in phenomenological applications. 
It is convenient to quote the results in three groups in which the polarized TMDs have structurally similar expressions. We can express $g_{1}^{q}\left(x, p_{T}\right), g_{L}^{\perp q}\left(x, p_{T}\right), h_{T}^{\perp q}\left(x, p_{T}\right)$ (which exhibit nodes) and $h_{T}^{\perp q}\left(x, p_{T}\right)$ in terms of transversity as

$$
\begin{gathered}
g_{1}^{q}\left(x, p_{T}\right) \stackrel{\mathrm{CPM}}{=}\left[1-\frac{p_{T}^{2}}{x^{2} M^{2}}\right] h_{1}^{q}\left(x, p_{T}\right), \\
g_{L}^{\perp q}\left(x, p_{T}\right) \stackrel{\mathrm{CPM}}{=} \frac{1}{x}\left[1-\frac{p_{T}^{2}}{x^{2} M^{2}}\right] h_{1}^{q}\left(x, p_{T}\right), \\
h_{T}^{q}\left(x, p_{T}\right) \stackrel{\mathrm{CPM}}{=}-\frac{1}{x}\left[1-\frac{p_{T}^{2}}{x^{2} M^{2}}\right] h_{1}^{q}\left(x, p_{T}\right), \\
h_{T}^{\perp q}\left(x, p_{T}\right) \stackrel{\mathrm{CPM}}{=} \frac{1}{x}\left[1+\frac{p_{T}^{2}}{x^{2} M^{2}}\right] h_{1}^{q}\left(x, p_{T}\right) .
\end{gathered}
$$

The TMDs $g_{T}^{q}\left(x, p_{T}\right)$ and $h_{L}^{q}\left(x, p_{T}\right)$ are expressed in terms of transversity (for $m=0$ ) as follows

$$
\begin{aligned}
& g_{T}^{q}\left(x, p_{T}\right) \stackrel{\mathrm{CPM}}{=}\left[\frac{p_{T}^{2}}{x^{2} M^{2}}\right] h_{1}^{q}\left(x, p_{T}\right), \\
& h_{L}^{q}\left(x, p_{T}\right) \stackrel{\mathrm{CPM}}{=}\left[\frac{2 p_{T}^{2}}{x^{2} M^{2}}\right] h_{1}^{q}\left(x, p_{T}\right) .
\end{aligned}
$$

Notice the relation $h_{L}^{q}\left(x, p_{T}\right) \stackrel{\mathrm{CPM}}{=} 2 g_{T}^{q}\left(x, p_{T}\right)$, which holds for $m=0$. Finally, the Mulders-Kotzinian TMDs $g_{1 T}^{\perp q}\left(x, p_{T}\right)$ and $h_{1 L}^{\perp q}\left(x, p_{T}\right)$, pretzelosity $h_{1 T}^{\perp q}\left(x, p_{T}\right)$, and twist-3 TMD $g_{T}^{\perp q}\left(x, p_{T}\right)$ are expressed in terms of transversity as

$$
\begin{aligned}
& g_{1 T}^{\perp q}\left(x, p_{T}\right) \stackrel{\mathrm{CPM}}{=} \frac{2}{x} h_{1}^{q}\left(x, p_{T}\right), \\
& h_{1 L}^{\perp q}\left(x, p_{T}\right) \stackrel{\mathrm{CPM}}{=}-\frac{2}{x} h_{1}^{q}\left(x, p_{T}\right), \\
& h_{1 T}^{\perp q}\left(x, p_{T}\right) \stackrel{\mathrm{CPM}}{=}-\frac{2}{x^{2}} h_{1}^{q}\left(x, p_{T}\right), \\
& g_{T}^{\perp q}\left(x, p_{T}\right) \stackrel{\mathrm{CPM}}{=} \frac{2}{x^{2}} h_{1}^{q}\left(x, p_{T}\right) .
\end{aligned}
$$

It will be interesting to test these CPM predictions in future, when more about TMDs will be known. These relations can also be investigated in other models. It would be interesting to assess in this way whether the relations (29)-(30) are supported by other quark models and, if so, within which accuracy.

\section{NUMERICAL RESULTS}

In this section we show the numerical results. After a brief review how the covariant functions $\mathcal{G}^{a}(p P)$ and $\mathcal{H}^{a}(p P)$ are obtained from the input PDFs $f_{1}^{a}(x)$ and $g_{1}^{a}(x)$, we present predictions for unpolarized and polarized PDFs or transverse moments of TMDs, and compare to parametrizations where available.

\section{A. Covariant functions and input PDFs}

The covariant functions $\mathcal{G}^{a}(p P)$ for $a=u, d, \bar{u}, \bar{d}, \ldots$ are uniquely determined from, respectively, $f_{1}^{a}(x)$ and $g_{1}^{a}(x)$. With the notation $\mathcal{G}^{q}\left(p^{0}\right)$ and $\mathcal{H}^{q}\left(p^{0}\right)$ in the nucleon rest frame, the relations are given by $[57,61]$ (see also [120])

$$
\begin{aligned}
\left.\mathcal{G}^{q}\left(p^{0}\right)\right|_{p^{0}=\frac{1}{2} x M}= & -\frac{1}{\pi M^{3}} \frac{d}{d x}\left[\frac{f_{1}^{q}(x)}{x}\right], \\
\left.\mathcal{H}^{q}\left(p^{0}\right)\right|_{p^{0}=\frac{1}{2} x M}= & \frac{1}{\pi M^{3} x^{2}}\left[2 \int_{x}^{1} \frac{d y}{y} g_{1}^{q}(y)+3 g_{1}^{q}(x)\right. \\
& \left.-x \frac{d}{d x}\left[\frac{g_{1}^{q}(x)}{x}\right]\right] .
\end{aligned}
$$

Several comments are in order. First, the model relates different TMDs obeying different evolution equations to the same covariant functions. Therefore, a scale must be chosen at which the covariant functions in (31) are determined. The choice of this scale is part of the modeling. The renormalization scale, which is not indicated in (31) for brevity, must be chosen large enough for the partonic picture to be justified, but is otherwise not fixed. In this work we choose $\mu^{2}=2.5 \mathrm{GeV}^{2}$ which is a convenient scale because many extractions of TMDs from SIDIS data have been performed at comparable scales. Second, in the case of TMDs we strictly speaking deal with a double-scale problem, and the choice of both scales is part of the model. The second scale $\zeta$, associated with the removal of rapidity divergences, can be also chosen to be $\zeta=2.5 \mathrm{GeV}^{2}$. The dependence on this second scale is governed by the CSS evolution equations. Third, an important feature of the parton model is the partonic interpretation. In QCD the partonic interpretation is strictly speaking only justified at leading-order (LO) (and for PDFs of the nucleon but, e.g., not for nuclei [159]). For our calculations we therefore choose LO parametrizations. In order to investigate the dependence on the chosen input parametrization, we use several parametrizations for respectively $f_{1}^{a}(x)$ and $g_{1}^{a}(x)$.

The parametrizations used in this work are shown in Fig. 1: GRV'98 [160], MRST'98 [161], MSTW'09 [162] for $f_{1}^{q}(x)$, and standard and valence scenarios of GRSV'00 [163], LSS'05 [164] for $g_{1}^{q}(x)$. More recent parametrizations are available, e.g., [165-169] for unpolarized PDFs, or [170-174] for helicity PDFs, see Ref. [175] for a review. The reason why for our purposes the earlier parametrizations [160-164] are preferable is because all more recent 

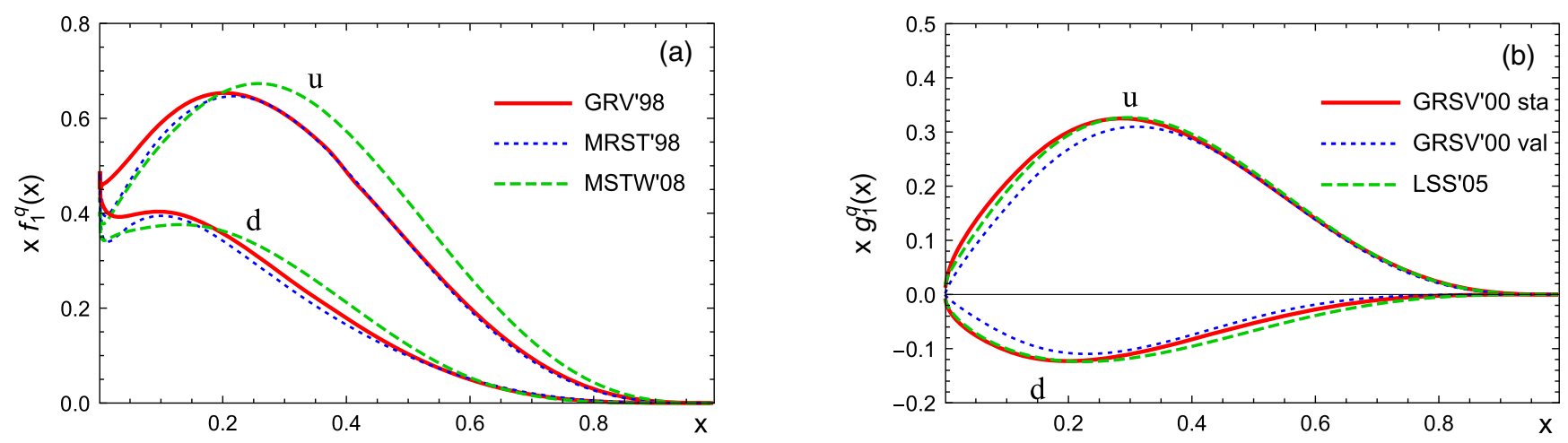

FIG. 1. The input PDFs used in this work for the model calculations as functions of $x$ at the scale $2.5 \mathrm{GeV}^{2}$. (a) LO parametrizations of the unpolarized PDFs $x f_{1}^{q}(x)$ from GRV'98 [160], MRST'98 [161], MSTW'09 [162]. (b) LO parametrizations of the helicity PDFs $x g_{1}^{q}(x)$ from GRSV'00 standard and valence scenario (see text) [163], and LSS'05 [164].

helicity PDF parametrizations were performed at next-toleading order, and [163,164] are among the last LO helicity parametrizations (recall that the use of LO parametrization is preferable in our partonic approach). The GRSV'00 [163] parametrizations of $g_{1}^{q}(x)$ were obtained using GRV'98 [160] for $f_{1}^{q}(x)$, while LSS'05 parametrizations of $g_{1}^{q}(x)$ were obtained using $f_{1}^{q}(x)$ from MRST'98 [161]. In addition, we use also the more recent MSTW'09 [162] parametrizations for $f_{1}^{q}(x)$.

Over the last two decades the parametrizations of quark PDFs $f_{1}^{q}(x)$ and $g_{1}^{q}(x)$ for $q=u, d$ have changed moderately, unlike especially antiquark helicity parametrizations which changed significantly due to recent data and may change further due to future Drell-Yan data from Brookhaven National Lab or the Electron-Ion Collider [176].

\section{B. The $p_{T}$ dependence of TMDs}

The $p_{T}$ dependence of twist-2 TMDs was discussed in [61]. ${ }^{2}$ The new results for twist-3 TMDs derived here have very similar $p_{T}$ dependencies, and we can refrain from discussing them in this work. Instead, we will content ourselves with briefly reviewing the main features of the $p_{T}$ dependencies of TMDs in this section.

It is remarkable that the $p_{T}$ dependencies of $f_{1}^{q}\left(x, p_{T}\right)$ and other unpolarized T-even TMDs are uniquely predicted from the input PDF $f_{1}^{q}(x)$. Similarly, the $p_{T}$ dependencies of $g_{1}^{q}\left(x, p_{T}\right)$ and other polarized T-even TMDs are uniquely

\footnotetext{
${ }^{2}$ At this occasion we would like to make a correction regarding [61]. The analytical results in [61] are correct (except for obvious misprints in Eq. (18) where it should be $\xi \frac{d g_{1}^{q}(\xi)}{d \xi}$ on the right-hand side instead of $x \frac{d q_{1}^{q}(\xi)}{d \xi}$ and Eq. (19) of [61] where it should be $M^{2}$ in the denominator instead of $M^{3}$ ). Also the numerical results in [61] were correctly computed. But due to an unfortunate plotting mistake the Figs. 1-4 of [61] show exactly half of the correct results for all the TMDs. None of the conclusions of [61] is affected by this mistake.
}

predicted from the input $\operatorname{PDF} g_{1}^{q}(x)$. This is possible due to the strong model assumption of on shell quarks which leads to the 3D rotational symmetry in nucleon rest frame encoded in the covariant functions $\mathcal{G}^{q}\left(p^{0}\right), \mathcal{H}^{q}\left(p^{0}\right)$ with $p^{0}=|\vec{p}|$ for massless quarks. This 3D symmetry connects longitudinal and transverse parton motion.

The TMDs have finite support for $0<p_{T}<$ $M \sqrt{x(1-x)}$ and vanish outside this range [53]. The covariant functions are nonzero only for $0<p^{0}<\frac{1}{2} M$ for massless partons due to $0<x<1$ in Eq. (31). In this work we will restrict ourselves to the discussion of model predictions after the transverse momenta are integrated out, and present results for PDFs like $h_{1}^{q}(x)$ or $g_{T}^{q}(x)$ or transverse moments of TMDs, defined, e.g., as

$$
h_{1 T}^{\perp(n) q}(x)=\int d^{2} p_{T}\left(\frac{p_{T}^{2}}{2 M^{2}}\right)^{n} h_{1 T}^{\perp q}\left(x, p_{T}\right)
$$

and analog for other TMDs. It is convenient to describe the respective structure functions in terms of such transverse moments. For instance in SIDIS the functions $h_{1}^{q}(x)$, $h_{1 L}^{\perp(1) q}(x), h_{1 T}^{\perp(2) q}(x)$ enter [156]. In the following we show, unless otherwise stated, those functions (PDFs or certain transverse moments) that are relevant for phenomenological applications. Where possible we will test the model predictions by comparing to parametrizations.

\section{Predictions for unpolarized TMDs}

In the unpolarized sector there are only three T-even TMDs: the twist $2 f_{1}^{q}\left(x, p_{T}\right)$ which is input in the model, and the twist- $3 f^{\perp q}\left(x, p_{T}\right)$ and $e^{q}\left(x, p_{T}\right)$. Both TMDs are related in the CPM to $f_{1}^{q}\left(x, p_{T}\right)$ according to Eqs. (29a) and (29b).

No relation between $f_{1}^{q}\left(x, p_{T}\right)$ and $f^{\perp q}\left(x, p_{T}\right)$ exists in QCD due to the appearance of the function $\tilde{f}^{\perp}\left(x, p_{T}\right)$. In general in quark models the tilde terms are also nonzero due to nontrivial quark-model interactions. It is therefore 

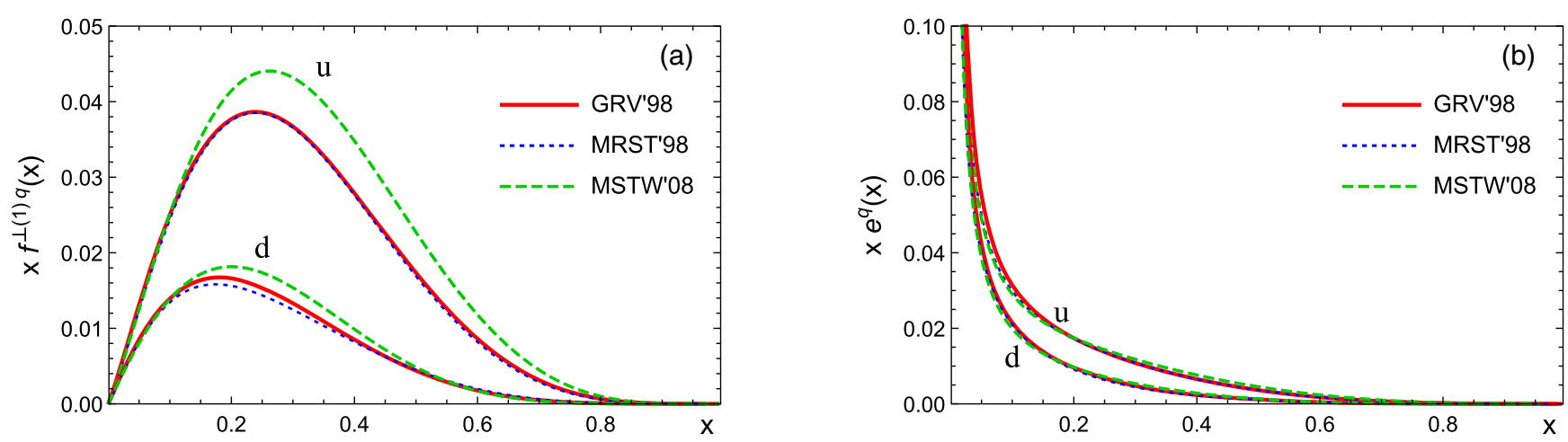

FIG. 2. Predictions for (a) $x f^{\perp(1) q}(x)$ and (b) $x e^{q}(x)$ as functions of $x$ at the scale $2.5 \mathrm{GeV}^{2}$ from the CPM according to Eqs. (29a) and (29b) using the unpolarized LO input PDFs shown in Fig. 1(a).

interesting that the relation (29) holds in lightfront constituent quark model and chiral quark soliton model even though both models exhibit nontrivial model interactions, encoded in the nonperturbative light front wave functions of the former or provided by the strong chiral interactions of the latter model [100].

The CPM prediction for $x f^{\perp(1) q}(x)$ is shown in Fig. 2(a), and corresponds exactly to the estimate for this TMD obtained in [156] on the basis of the WW-type approximation. The result in Fig. 2(a) shows that $x f^{\perp(1) q}(x)$ is sizable.

The situation is different for $x e^{q}(x)$ which vanishes if we neglect quark mass effects. In order to show a nonzero result we assume $m_{q}=5 \mathrm{MeV}$ for both $u$ and $d$ flavor. As expected, the $x e^{q}(x)$ resulting from Eq. (29a) is very small. Assuming TMD factorization at twist-3 level [177], $e^{q}\left(x, p_{T}\right)$ contributes to observables, e.g., in SIDIS with the prefactor $M / Q$ [4] where $Q$ is the hard scale of the process. The contribution of the mass term in $e^{q}\left(x, p_{T}\right)$ is therefore effectively proportional to $m_{q} / Q$ and can be safely neglected in many phenomenological applications [156]. Only at extremely small $x \lesssim m_{q} / Q$ could the mass term contribution to $x e^{q}(x)$ become important. This kinematics will be accessible at the Electron-Ion Collider [1]. However, the discussion of TMDs at small $x$ is more adequately addressed in the approach of Refs. [178,179] which, to the best of our knowledge, has not yet been applied to subleading twist. It is interesting to remark that, e.g., in the light front constituent quark model the relation (29a) is also valid, but $e^{q}(x)$ in nevertheless sizable, because that model operates at a low hadronic scale $\mu<1 \mathrm{GeV}$ where the effective quark degrees of freedom have a constituent quark mass of about $300 \mathrm{MeV}$ [100].

No model-independent extractions of these twist-3 TMDs are currently available. In the case of $e^{q}(x)$ very first (and model-dependent) extractions were reported in Refs. [180,181]. For further model studies of $f^{\perp(1) q}(x)$ and $e^{q}(x)$, including the interesting possibility of a singular $\delta(x)$ contribution to $e^{q}(x)$, which is beyond our partonic approach, we refer to Refs. [60,67,72,73,89-92,102,109,
110,182-186]. Noteworthy is the partonic interpretation of the pure twist- 3 contribution to $e^{q}(x)$ in terms of transverse forces experienced by quarks in DIS [187].

\section{Results for transversity and pretzelosity}

Next we turn our attention to polarized TMDs starting the discussion with the twist- 2 transversity and pretzelosity which can be compared to available parametrizations $[45,46]$.

In Figs. 3(a) and 3(b) we compare the model predictions for $x h_{1}^{q}(x)$ to the recent JAM'20 parametrization [45]. The CPM describes the sign and magnitude of the transversity quark distributions well. For larger $x \gtrsim 0.2$ the quantitative agreement is very good and the model results are close to or within the $1-\sigma$ region of the extraction [45]. At smaller $x \lesssim 0.2$ the model has a tendency to overestimate the JAM'20 parametrization for $u$ and $d$ flavors. The uncertainty of the JAM'20 parametrization [45] is still very large. For instance the $d$-quark transversity is compatible with zero within the $1-\sigma$ uncertainty of the extraction. Future data will constrain more strongly the extractions and allow us to test the model predictions for $h_{1}^{q}(x)$ more rigorously. The CPM is in good qualitative agreement with other model calculations [60,67,72,73,75,76,80,81,84,87, 88,95,101,104,111,188] and lattice QCD [115,116].

In Figs. 3(c) and 3(d) we compare the model predictions for $x h_{1 T}^{\perp(1) q}(x)$ to the LP'15 fit [46] (where the (1)-moment was extracted, though in phenomenological applications $[156,157]$ the (2)-moment of pretzelosity enters naturally). The present data on the azimuthal asymmetry related to pretzelosity are compatible with zero, which is reflected by the uncertainty band of the LP' 15 parametrization for $u$ and $d$ flavors [46]. The best fit of LP' 15 has opposite sign to the CPM which should be not too disturbing considering the large uncertainties of the fit. The CPM results agree with other models [60,72,73,76-81,95]. More precise future data are needed to test the model predictions for pretzelosity. This TMD is of interest because it is related to deviations of the nucleon's transverse spin distribution 

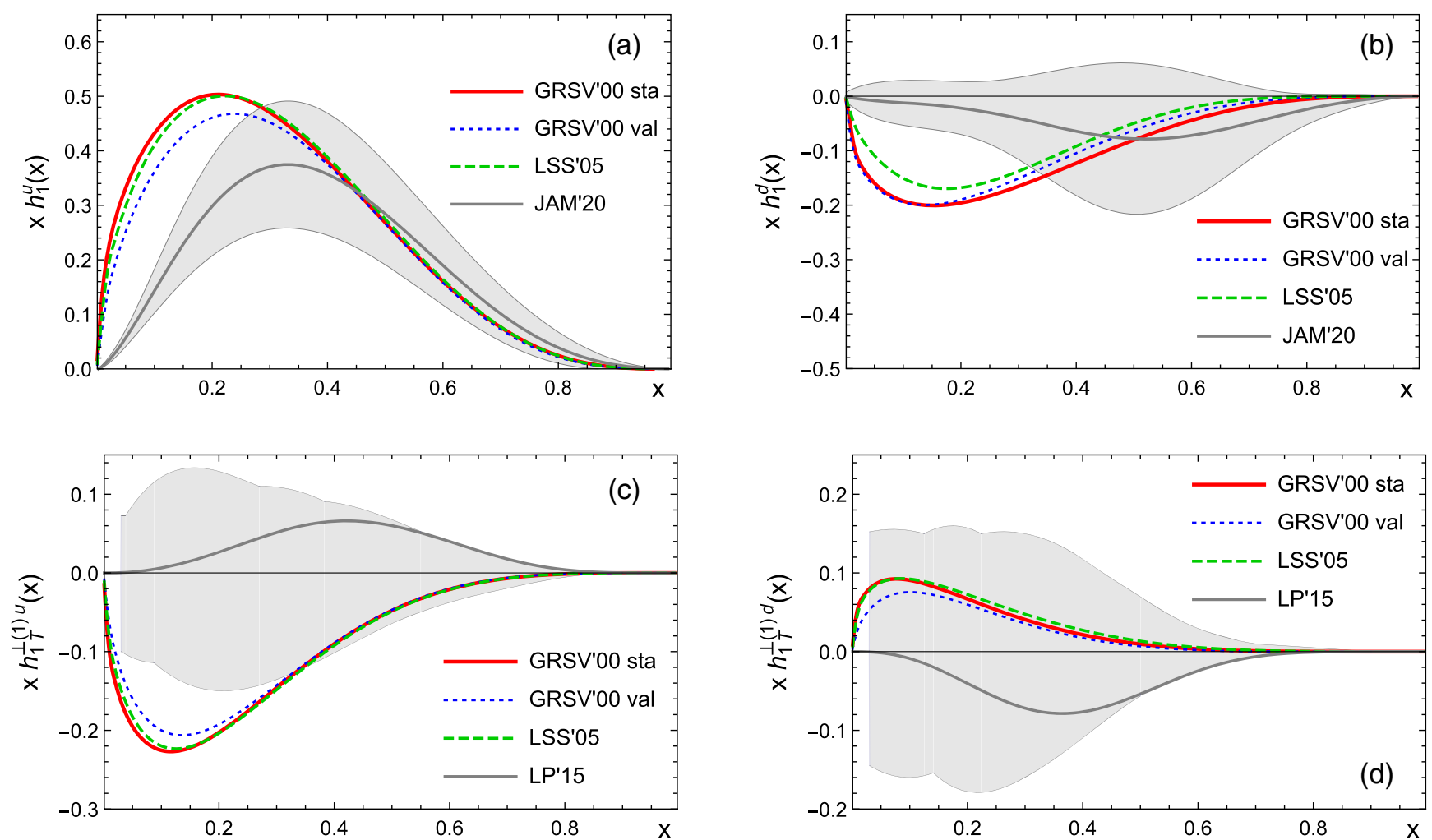

FIG. 3. Model predictions for $x h_{1}^{q}(x)$, (a) and (b), and $x h_{1 T}^{\perp(1) q}(x),(\mathrm{c})$ and (d), as functions of $x$ at the scale $2.5 \mathrm{GeV}^{2}$ obtained using the helicity LO input PDFs from Fig. 1(b) in comparison to, respectively, the JAM'20 [45] and LP'15 [46] parametrizations.

from spherical symmetry [189] and, in certain models, to quark orbital momentum $[70,77,122]$.

\section{E. Kotzinian-Mulders functions}

In this section we continue the discussion of polarized twist-2 TMDs for which currently no extractions are available, the Kotzinian-Mulders functions $g_{1 T}^{\perp(1) q}(x)$ and $h_{1 L}^{\perp(1) q}(x)$. In the CPM and in many other models these TMDs are related to each other by the Eq. (21). For clarity we nevertheless show the results for both TMDs in separate figures.

In Fig. 4(a) we show the model results for $x g_{1 T}^{\perp(1) q}(x)$. The model supports the WW-type relation (25), which was used in Ref. [156]. This means that in Ref. [156] exactly the same predictions as presented in Fig. 4(a) were used for this TMD and shown to be compatible with the data currently available on this TMD. This means that the CPM model prediction for $g_{1 T}^{\perp(1) q}(x)$ is also compatible with the currently available SIDIS data. One should add
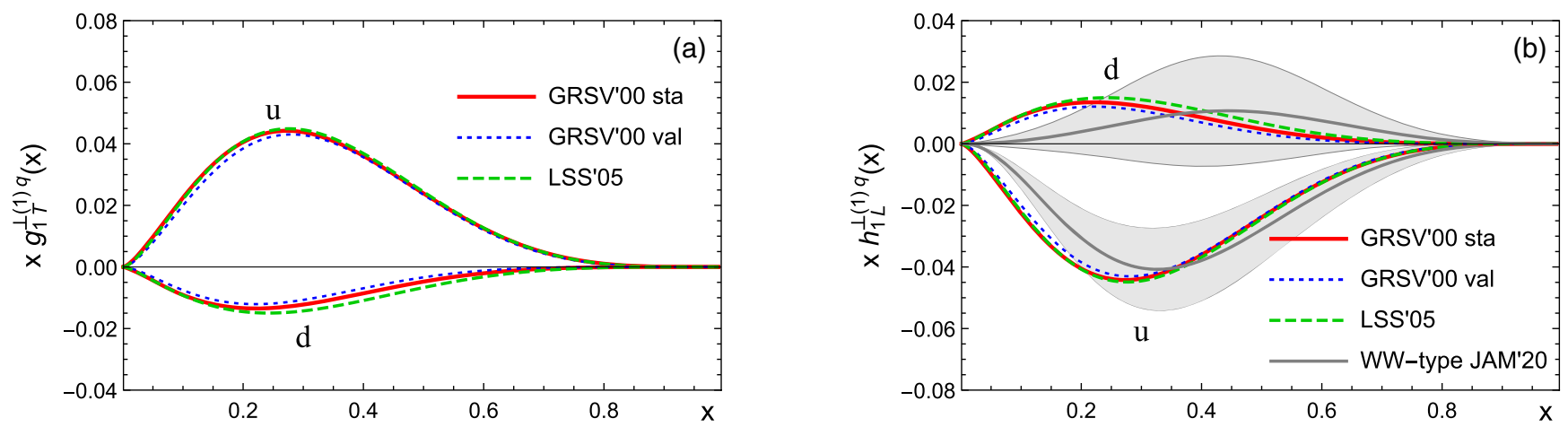

FIG. 4. Model predictions for (a) $x g_{1 T}^{\perp(1) q}(x)$ and (b) $x h_{1 L}^{\perp(1) q}(x)$ for $q=u, d$ as functions of $x$ at the scale $2.5 \mathrm{GeV}^{2}$ obtained using the helicity LO input PDFs from Fig. 1(b). For comparison we show in (b) the estimate for $x h_{1 L}^{\perp(1) q}(x)$ from [156] based on the WW-type approximation and JAM'20 transversity parametrization [45]. 

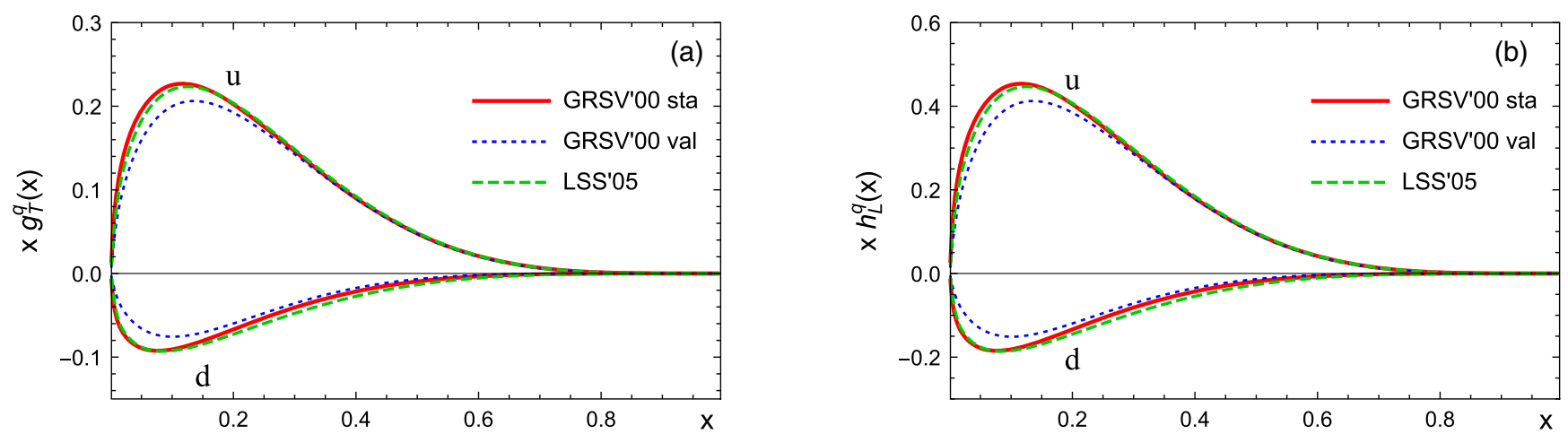

FIG. 5. Model predictions for the twist-3 PDFs (a) $x g_{T}^{q}(x)$ and (b) $x h_{L}^{q}(x)$ as functions of $x$ at the scale $2.5 \mathrm{GeV}^{2}$ obtained using the helicity LO input PDFs from Fig. 1(b).

that the existing data on the pertinent SIDIS asymmetry have sizable error bars and this test of the model is at the current stage rather qualitative. However, more precise future data will allow us to make more quantitative tests of the model.

In Fig. 4(b) we plot the model results for $x h_{1 L}^{\perp(1) q}(x)$. For comparison we show also the estimate for this TMD from [156], which is based on the WW-type approximation (25b) and JAM'20 $h_{1}^{q}(x)$ parametrization [45]. Though the CPM supports Eq. (25b), the comparison in Fig. 4(b) is nevertheless interesting: in our model this TMD is ultimately obtained from the input helicity PDF. In contrast to this the WW-type-based prediction for $h_{1 L}^{\perp(1) q}(x)$ from [156] is based on (25b) and transversity as input. Thus, these are two different ways of making predictions for this Kotzinian-Mulders function and the good agreement of the two results constitutes a consistency check in the sense that the CPM practically supports numerical estimates of this TMD based on the WW-type approximations [156].

\section{F. Predictions for polarized twist-3 PDFs and TMDs}

In this section we discuss polarized twist-3 TMDs beginning with $g_{T}^{q}(x)$ which is accessible in polarized DIS making it the only well-constrained twist-3 function. In the CPM the WW approximation (24) holds exactly.
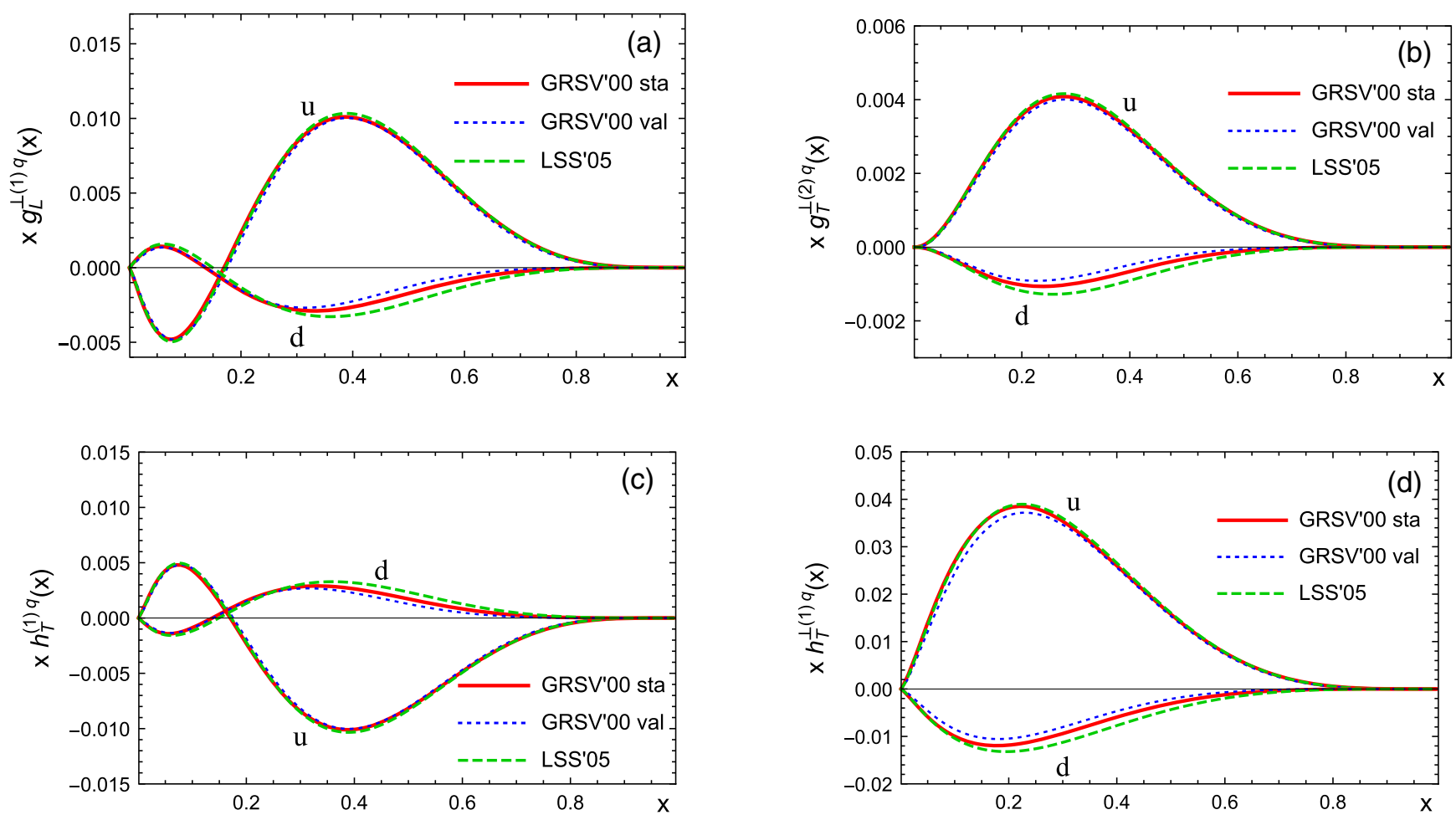

FIG. 6. Model predictions for the twist-3 polarized TMDs (a) $x g_{L}^{\perp(1) q}(x)$, (b) $x g_{T}^{\perp(2) q}(x)$, (c) $x h_{T}^{(1) q}(x)$, (d) $x h_{T}^{\perp(1) q}(x)$ as functions of $x$ at the scale $2.5 \mathrm{GeV}^{2}$ obtained using the helicity LO input PDFs from Fig. 1(b). 
The model prediction is shown in Fig. 5(a). Interestingly, the Mellin moments of the pure twist- 3 contribution $\tilde{g}_{T}^{q}(x)$ were shown in instanton vacuum calculations to be strongly suppressed by powers of the instanton packing fraction [134]. Subsequently the smallness of the $\tilde{g}_{T}^{q}(x)$ contribution to $g_{T}^{q}(x)$ was confirmed in experiments [139-141] and lattice QCD studies [137,138]. These theoretical and experimental results have been a main motivation for exploring the possibility of WW- and WW-type approximations $[39,129,156,157]$ though it cannot be excluded that $\tilde{g}_{T}^{q}(x)$ might be sizable in certain (so far experimentally unexplored or poorly unconstrained) $x$ regions [155]. The smallness of $\tilde{g}_{T}^{q}(x)$ provides important support also for the CPM, and played an important role in the development of this model [55].

The only further polarized twist-3 collinear PDF is $h_{L}^{q}(x)$ which, however, is chirally odd and not accessible in DIS. Consequently almost nothing is known phenomenologically about this PDF. Interestingly, also in this case the pure twist- 3 contribution $\tilde{h}_{L}^{q}(x)$ is also suppressed in the instanton vacuum [135]. In the CPM the corresponding WW relation (24b) is exact. The model prediction for $x h_{L}^{q}(x)$ is shown in Fig. 5(b). This function contributes to single spin asymmetries in SIDIS [3] with several other unknown twist-3 TMDs and fragmentation functions such that phenomenological information on this TMD is difficult to obtain $[151,152]$. Of interest is the CPM prediction $h_{L}^{q}(x)=2 g_{T}^{q}(x)$. It will be interesting to see if this prediction will be supported phenomenologically.

Finally, we show the predictions for $x g_{L}^{\perp(1) q}(x)$ in Fig. 6(a), $x g_{T}^{\perp(2) q}(x)$ in Fig. 6(b), $x h_{T}^{(1) q}(x)$ in Fig. 6(c), $x h_{T}^{\perp(1) q}(x)$ in Fig. 6(d). These transverse moments are rather small, and the contributions of these TMDs to the SIDIS structure functions can be expected to be small. Currently nothing is known about those TMDs from phenomenology [156]. The model predicts the relation $g_{L}^{\perp q}\left(x, p_{T}\right)=-h_{T}^{q}\left(x, p_{T}\right)$, which will be interesting to test experimentally, and both TMDs exhibit a node around $x \approx 0.15$, which is also observed in the bag model [72]. The same relation between $g_{L}^{\perp q}\left(x, p_{T}\right)$ and $h_{T}^{q}\left(x, p_{T}\right)$ holds also in the spectator and bag model [72,73], which may hint at a possible more general underlying quark model symmetry responsible for such relations among twist-3 TMDs. It would be interesting to investigate this point in more detail.

\section{CONCLUSIONS}

In this work we have generalized the CPM that was originally formulated to describe PDFs accessible in DIS through an intuitive modeling of the hadronic tensor. We have shown that the new formulation of the CPM allows one to reproduce all results for the T-even twist-2 TMDs $f_{1}^{q}$, $g_{1}^{q}, h_{1}^{q}, g_{1 T}^{\perp q}, h_{1 L}^{\perp q}, h_{1 T}^{\perp q}$, and the twist-3 $g_{T}^{q}$ known from prior studies. The advantage of the new formulation is that it allows one to evaluate systematically quark correlators in the CPM. We have demonstrated this by deriving the model expressions for all twist-3 T-even TMDs.

We have checked the consistency of the model by showing that the QCD equations-of-motion relations are valid in the CPM with tilde-terms consistently being zero which is to be expected in a parton model approach. The model also complies with Lorentz-invariance relations, which are valid in quark models that respect Lorentz symmetry but lack explicit gauge field degrees of freedom. We have investigated the relations among TMDs in the CPM. Some of these relations were known from prior studies in other models, but most of them are specific to our model. The relations among TMDs may constitute one of the most interesting predictions of the CPM and they will allow one to test the underlying model concepts quantitatively in future when more information about TMDs will become available.

We presented numerical predictions for the T-even TMDs and compared to studies in other models or lattice QCD, and confronted them with TMD parametrizations available for transversity and pretzelosity (the latter with very large uncertainties). No extractions are available for other TMDs and the model predictions await phenomenological tests in those cases. One interesting advantage is that the results from the CPM refer to a high renormalization scale where the partonic interpretation may be assumed to be valid, while to the best of our knowledge the results from other quark model approaches refer to very low hadronic scales $\mu<1 \mathrm{GeV}$ [67-111].

The new formulation of the CPM may have further interesting applications going far beyond the computation of the twist-3 TMDs presented in this work. It would be interesting to introduce a consistent modeling of off-shellness effects. This would allow one to compute tilde functions and perhaps also describe T-odd TMDs. Another interesting application could be the extension of the model to antiquark correlators or gluon correlators. These aspects will be addressed in future studies.

\section{ACKNOWLEDGMENTS}

We wish to thank E. Leader, D. B. Stamenov, and W. Vogelsang for providing us with the LO parametrizations of the Refs. [163,164] and Barbara Pasquini for helpful discussions. The work of S. B. and P.S. was supported by the National Science Foundation under the Award No. 1812423. The work of P. Z. was supported by Grant No. LTT17018 of the MEYS (Czech Republic).

Note added in proof.-We are sad to have to announce that Anatoli Vasilievich Efremov passed away on January 1st, 2021 at the age of 87 years. 


\section{APPENDIX: MODEL RESULTS FOR TMDS IN COMPACT NOTATION}

In order to have a better overview, it is convenient to introduce the compact notation for the integration measures

$$
\begin{aligned}
\left\{\mathrm{d} p_{\mathcal{G} q}^{1}\right\} & =\frac{\mathrm{d} p^{1}}{p^{0}} \frac{\mathcal{G}^{q}\left(p^{0}\right)}{\left(p^{0}+m\right)} \delta\left(x-\frac{p^{0}+p^{1}}{M}\right), \\
\left\{\mathrm{d} p_{\mathcal{H} q}^{1}\right\} & =\frac{\mathrm{d} p^{1}}{p^{0}} \frac{\mathcal{H}^{q}\left(p^{0}\right)}{\left(p^{0}+m\right)} \delta\left(x-\frac{p^{0}+p^{1}}{M}\right) .
\end{aligned}
$$

Then the model results for the leading twist T-even TMDs can be summarized as follows

$$
\begin{aligned}
f_{1}^{q}\left(x, p_{T}\right) & =\int\left\{\mathrm{d} p_{\mathcal{G} q}^{1}\right\}\left[M x\left(p^{0}+m\right)\right], \\
g_{1}^{q}\left(x, p_{T}\right) & =\int\left\{\mathrm{d} p_{\mathcal{H} q}^{1}\right\}\left[M x\left(p^{0}+m\right)-\vec{p}_{T}^{2}\right], \\
g_{1 T}^{\perp q}\left(x, p_{T}\right) & =\int\left\{\mathrm{d} p_{\mathcal{H} q}^{1}\right\}[M(M x+m)], \\
h_{1}^{a}\left(x, p_{T}\right) & =\int\left\{\mathrm{d} p_{\mathcal{H} q}^{1}\right\}\left[M x\left(p^{0}+m\right)-\frac{\vec{p}_{T}^{2}}{2}\right], \\
h_{1 L}^{\perp q}\left(x, p_{T}\right) & =\int\left\{\mathrm{d} p_{\mathcal{H} q}^{1}\right\}[-M(M x+m)], \\
h_{1 T}^{\perp q}\left(x, p_{T}\right) & =\int\left\{\mathrm{d} p_{\mathcal{H} q}^{1}\right\}\left[-M^{2}\right],
\end{aligned}
$$

and the twist-3 T-even TMDs are given by

$$
\begin{array}{r}
f^{\perp q}\left(x, p_{T}\right)=\int\left\{\mathrm{d} p_{\mathcal{G} q}^{1}\right\}\left[M\left(p^{0}+m\right)\right], \\
e^{q}\left(x, p_{T}\right)=\int\left\{\mathrm{d} p_{\mathcal{G} q}^{1}\right\}\left[m\left(p^{0}+m\right)\right], \\
g_{L}^{\perp q}\left(x, p_{T}\right)=\int\left\{\mathrm{d} p_{\mathcal{H} q}^{1}\right\}\left[M\left(M x-p^{0}\right)\right], \\
g_{T}^{q}\left(x, p_{T}\right)=\int\left\{\mathrm{d} p_{\mathcal{H} q}^{1}\right\}\left[m\left(p^{0}+m\right)+\frac{1}{2} \vec{p}_{T}^{2}\right], \\
g_{T}^{\perp q}\left(x, p_{T}\right)=\int\left\{\mathrm{d} p_{\mathcal{H} q}^{1}\right\}\left[M^{2}\right], \\
h_{L}^{q}\left(x, p_{T}\right)=\int\left\{\mathrm{d} p_{\mathcal{H} q}^{1}\right\}\left[m\left(p^{0}+m\right)+\vec{p}_{T}^{2}\right], \\
h_{T}^{q}\left(x, p_{T}\right)=\int\left\{\mathrm{d} p_{\mathcal{H} q}^{1}\right\}\left[M\left(p^{0}-M x\right)\right], \\
h_{T}^{\perp q}\left(x, p_{T}\right)=\int\left\{\mathrm{d} p_{\mathcal{H} q}^{1}\right\}\left[M\left(p^{0}+m\right)\right] .
\end{array}
$$

The model expressions can be rewritten by exploring the on-shell-ness of partons which gives rise to identities like $\left(p^{0}+p^{1}\right)\left(p^{0}+m\right)-\frac{1}{2} p_{T}^{2}=\frac{1}{2}(x M+m)^{2}$ and can be used to express transversity, e.g., as $h_{1}^{q}\left(x, p_{T}\right)=$ $\int\left\{\mathrm{d} p_{\mathcal{H} q}^{1}\right\} \frac{1}{2}(x M+m)^{2}$.
[1] A. Accardi et al., Eur. Phys. J. A 52, 268 (2016); D. Boer et al., arXiv:1108.1713.

[2] A. Kotzinian, Nucl. Phys. B441, 234 (1995).

[3] P. Mulders and R. Tangerman, Nucl. Phys. B461, 197 (1996).

[4] A. Bacchetta, M. Diehl, K. Goeke, A. Metz, P. J. Mulders, and M. Schlegel, J. High Energy Phys. 02 (2007) 093.

[5] A. Metz and A. Vossen, Prog. Part. Nucl. Phys. 91, 136 (2016).

[6] D. Boer and P. J. Mulders, Phys. Rev. D 57, 5780 (1998).

[7] S. Arnold, A. Metz, and M. Schlegel, Phys. Rev. D 79, 034005 (2009).

[8] J. C. Collins and D. E. Soper, Nucl. Phys. B193, 381 (1981); B213, 545(E) (1983).

[9] A. V. Efremov and O. V. Teryaev, Sov. J. Nucl. Phys. 36, 140 (1982).

[10] A. V. Efremov and O. V. Teryaev, Phys. Lett. 150B, 383 (1985).

[11] J. C. Collins, D. E. Soper, and G. F. Sterman, Nucl. Phys. B250, 199 (1985).
[12] J. W. Qiu and G. F. Sterman, Phys. Rev. Lett. 67, 2264 (1991).

[13] X. D. Ji, J. P. Ma, and F. Yuan, Phys. Rev. D 71, 034005 (2005).

[14] X. D. Ji, J. W. Qiu, W. Vogelsang, and F. Yuan, Phys. Lett. B 638, 178 (2006).

[15] X. Ji, J. w. Qiu, W. Vogelsang, and F. Yuan, Phys. Rev. D 73, 094017 (2006).

[16] J. Collins, Cambridge Monogr. Part. Phys Nucl. Phys. Cosmol. 32, 1 (2011).

[17] S. M. Aybat and T. C. Rogers, Phys. Rev. D 83, 114042 (2011).

[18] A. Bacchetta and A. Prokudin, Nucl. Phys. B875, 536 (2013).

[19] P. Sun and F. Yuan, Phys. Rev. D 88, 034016 (2013).

[20] M. G. Echevarria, A. Idilbi, Z. B. Kang, and I. Vitev, Phys. Rev. D 89, 074013 (2014).

[21] J. Collins and T. Rogers, Phys. Rev. D 91, 074020 (2015).

[22] J. Collins, L. Gamberg, A. Prokudin, T. C. Rogers, N. Sato, and B. Wang, Phys. Rev. D 94, 034014 (2016). 
[23] T. Gehrmann, T. Luebbert., and L. L. Yang, J. High Energy Phys. 06 (2014) 155.

[24] M. G. Echevarria, I. Scimemi, and A. Vladimirov, Phys. Rev. D 93, 054004 (2016).

[25] M. G. Echevarria, I. Scimemi, and A. Vladimirov, J. High Energy Phys. 09 (2016) 004.

[26] Y. Li and H. X. Zhu, Phys. Rev. Lett. 118, 022004 (2017).

[27] A. A. Vladimirov, Phys. Rev. Lett. 118, 062001 (2017).

[28] D. Gutiérrez-Reyes, I. Scimemi, and A. A. Vladimirov, Phys. Lett. B 769, 84 (2017).

[29] D. Gutierrez-Reyes, I. Scimemi, and A. A. Vladimirov, J. High Energy Phys. 07 (2018) 172.

[30] M. X. Luo, X. Wang, X. Xu, L. L. Yang, T. Z. Yang, and H. X. Zhu, J. High Energy Phys. 10 (2019) 083.

[31] M. X. Luo, T.Z. Yang, H. X. Zhu, and Y. J. Zhu, Phys. Rev. Lett. 124, 092001 (2020).

[32] M. A. Ebert, B. Mistlberger, and G. Vita, J. High Energy Phys. 09 (2020) 146.

[33] A. V. Efremov, K. Goeke, S. Menzel, A. Metz, and P. Schweitzer, Phys. Lett. B 612, 233 (2005).

[34] M. Anselmino, M. Boglione, U. D’Alesio, A. Kotzinian, F. Murgia, and A. Prokudin, Phys. Rev. D 71, 074006 (2005).

[35] W. Vogelsang and F. Yuan, Phys. Rev. D 72, 054028 (2005).

[36] J. C. Collins, A. V. Efremov, K. Goeke, S. Menzel, A. Metz, and P. Schweitzer, Phys. Rev. D 73, 014021 (2006).

[37] J. C. Collins, A. V. Efremov, K. Goeke, M. Grosse Perdekamp, S. Menzel, B. Meredith, A. Metz, and P. Schweitzer, Phys. Rev. D 73, 094023 (2006).

[38] M. Anselmino, M. Boglione, U. D’Alesio, A. Kotzinian, F. Murgia, A. Prokudin, and C. Turk, Phys. Rev. D 75, 054032 (2007).

[39] M. Anselmino, M. Boglione, U. D’Alesio, S. Melis, F. Murgia, and A. Prokudin, Phys. Rev. D 87, 094019 (2013).

[40] A. Signori, A. Bacchetta, M. Radici, and G. Schnell, J. High Energy Phys. 11 (2013) 194.

[41] M. Anselmino, M. Boglione, J. O. Gonzalez Hernandez, S. Melis, and A. Prokudin, J. High Energy Phys. 04 (2014) 005.

[42] Z. B. Kang, A. Prokudin, P. Sun., and F. Yuan, Phys. Rev. D 91, 071501 (2015).

[43] Z. B. Kang, A. Prokudin, P. Sun, and F. Yuan, Phys. Rev. D 93, 014009 (2016).

[44] Z. B. Kang, A. Prokudin, F. Ringer, and F. Yuan, Phys. Lett. B 774, 635 (2017).

[45] J. Cammarota, L. Gamberg, Zhong-Bo Kang, Joshua A. Miller, D. Pitonyak, A. Prokudin, T. C. Rogers, and N. Sato (Jefferson Lab Angular Momentum Collaboration), Phys. Rev. D 102, 054002 (2020).

[46] C. Lefky and A. Prokudin, Phys. Rev. D 91, 034010 (2015).

[47] J. C. Collins, Acta Phys. Pol. B 34, 3103 (2003).

[48] U. D’Alesio and F. Murgia, Prog. Part. Nucl. Phys. 61, 394 (2008).

[49] V. Barone, F. Bradamante, and A. Martin, Prog. Part. Nucl. Phys. 65, 267 (2010).

[50] C. A. Aidala, S. D. Bass, D. Hasch, and G. K. Mallot, Rev. Mod. Phys. 85, 655 (2013).
[51] H. Avakian, B. Parsamyan, and A. Prokudin, Riv. Nuovo Cimento 42, 1 (2019).

[52] M. Anselmino, A. Mukherjee, and A. Vossen, Prog. Part. Nucl. Phys. 114, 103806 (2020).

[53] P. Zavada, Phys. Rev. D 55, 4290 (1997).

[54] P. Zavada, Phys. Rev. D 65, 054040 (2002).

[55] P. Zavada, Phys. Rev. D 67, 014019 (2003).

[56] A. V. Efremov, O. V. Teryaev, and P. Zavada, Phys. Rev. D 70, 054018 (2004).

[57] P. Zavada, Eur. Phys. J. C 52, 121 (2007).

[58] A. V. Efremov, P. Schweitzer, O. V. Teryaev, and P. Zavada, Phys. Rev. D 80, 014021 (2009).

[59] P. Zavada, Phys. Rev. D 83, 014022 (2011).

[60] H. Avakian, A. V. Efremov, P. Schweitzer, O. V. Teryaev, F. Yuan, and P. Zavada, Mod. Phys. Lett. A 24, 2995 (2009).

[61] A. V. Efremov, P. Schweitzer, O. V. Teryaev, and P. Zavada, Phys. Rev. D 83, 054025 (2011).

[62] P. Zavada, Phys. Rev. D 85, 037501 (2012).

[63] P. Zavada, Phys. Rev. D 89, 014012 (2014).

[64] P. Zavada, Phys. Lett. B 751, 525 (2015).

[65] P. Zavada, arXiv:1911.12703.

[66] R. P. Feynman, Photon-Hadron Interactions (CRC Press, Reading, 1972).

[67] R. L. Jaffe and X. D. Ji, Nucl. Phys. B375, 527 (1992).

[68] F. Yuan, Phys. Lett. B 575, 45 (2003).

[69] A. Courtoy, F. Fratini, S. Scopetta, and V. Vento, Phys. Rev. D 78, 034002 (2008).

[70] H. Avakian, A. V. Efremov, P. Schweitzer, and F. Yuan, Phys. Rev. D 78, 114024 (2008).

[71] A. Courtoy, S. Scopetta, and V. Vento, Phys. Rev. D 79, 074001 (2009).

[72] H. Avakian, A. Efremov, P. Schweitzer, and F. Yuan, Phys. Rev. D 81, 074035 (2010).

[73] R. Jakob, P. J. Mulders, and J. Rodrigues, Nucl. Phys. A626, 937 (1997).

[74] L. P. Gamberg, G. R. Goldstein, and M. Schlegel, Phys. Rev. D 77, 094016 (2008).

[75] I. C. Cloet, W. Bentz, and A. W. Thomas, Phys. Lett. B 659, 214 (2008).

[76] A. Bacchetta, F. Conti, and M. Radici, Phys. Rev. D 78, 074010 (2008).

[77] J. She, J. Zhu, and B. Q. Ma, Phys. Rev. D 79, 054008 (2009).

[78] Z. Lu and I. Schmidt, Phys. Lett. B 712, 451 (2012).

[79] T. Maji, C. Mondal, D. Chakrabarti, and O. V. Teryaev, J. High Energy Phys. 01 (2016) 165.

[80] T. Maji and D. Chakrabarti, Phys. Rev. D 94, 094020 (2016).

[81] T. Maji and D. Chakrabarti, Phys. Rev. D 95, 074009 (2017).

[82] D. Diakonov, V. Petrov, P. Pobylitsa, M. V. Polyakov, and C. Weiss, Nucl. Phys. B480, 341 (1996).

[83] D. Diakonov, V. Petrov, P. Pobylitsa, M. Polyakov, and C. Weiss, Phys. Rev. D 56, 4069 (1997).

[84] L. P. Gamberg, H. Reinhardt, and H. Weigel, Phys. Rev. D 58, 054014 (1998).

[85] P. V. Pobylitsa, M. V. Polyakov, K. Goeke, T. Watabe, and C. Weiss, Phys. Rev. D 59, 034024 (1999).

[86] K. Goeke, P. V. Pobylitsa, M. V. Polyakov, P. Schweitzer, and D. Urbano, Acta Phys. Pol. B 32, 1201 (2001). 
[87] M. Wakamatsu, Phys. Lett. B 509, 59 (2001).

[88] P. Schweitzer, D. Urbano, M. V. Polyakov, C. Weiss, P. V. Pobylitsa, and K. Goeke, Phys. Rev. D 64, 034013 (2001).

[89] P. Schweitzer, Phys. Rev. D 67, 114010 (2003).

[90] M. Wakamatsu and Y. Ohnishi, Phys. Rev. D 67, 114011 (2003).

[91] Y. Ohnishi and M. Wakamatsu, Phys. Rev. D 69, 114002 (2004).

[92] C. Cebulla, J. Ossmann, P. Schweitzer, and D. Urbano, Acta Phys. Pol. B 39, 609 (2008).

[93] M. Wakamatsu, Phys. Rev. D 79, 094028 (2009).

[94] P. Schweitzer, M. Strikman, and C. Weiss, J. High Energy Phys. 01 (2013) 163.

[95] B. Pasquini, S. Cazzaniga, and S. Boffi, Phys. Rev. D 78, 034025 (2008).

[96] B. Pasquini and F. Yuan, Phys. Rev. D 81, 114013 (2010).

[97] S. Boffi, A. V. Efremov, B. Pasquini, and P. Schweitzer, Phys. Rev. D 79, 094012 (2009).

[98] C. Lorcé, B. Pasquini, and M. Vanderhaeghen, J. High Energy Phys. 05 (2011) 041.

[99] B. Pasquini and P. Schweitzer, Phys. Rev. D 83, 114044 (2011).

[100] C. Lorcé, B. Pasquini, and P. Schweitzer, J. High Energy Phys. 01 (2015) 103.

[101] S. Kofler and B. Pasquini, Phys. Rev. D 95, 094015 (2017).

[102] B. Pasquini and S. Rodini, Phys. Lett. B 788, 414 (2019).

[103] H. H. Matevosyan, W. Bentz, I. C. Cloet, and A. W. Thomas, Phys. Rev. D 85, 014021 (2012).

[104] Z. Alizadeh Yazdi, F. Taghavi-Shahri, F. Arash, and M. E. Zomorrodian, Phys. Rev. C 89, 055201 (2014).

[105] T. Maji, D. Chakrabarti, and A. Mukherjee, Phys. Rev. D 97, 014016 (2018).

[106] V. E. Lyubovitskij and I. Schmidt, Phys. Rev. D 102, 034011 (2020).

[107] R. Kundu and A. Metz, Phys. Rev. D 65, 014009 (2001).

[108] S. Meissner, A. Metz, and K. Goeke, Phys. Rev. D 76, 034002 (2007).

[109] A. Mukherjee, Phys. Lett. B 687, 180 (2010).

[110] A. Mukherjee and R. Korrapati, Mod. Phys. Lett. A 26, 2653 (2011).

[111] C. Mondal, S. Xu, J. Lan, X. Zhao, Y. Li, D. Chakrabarti, and J. P. Vary, Phys. Rev. D 102, 016008 (2020).

[112] P. Hägler, B. U. Musch, J. W. Negele, and A. Schäfer, Europhys. Lett. 88, 61001 (2009).

[113] B. U. Musch, P. Hägler, J. W. Negele, and A. Schäfer, Phys. Rev. D 83, 094507 (2011).

[114] B. U. Musch, P. Hägler, M. Engelhardt, J. W. Negele, and A. Schäfer, Phys. Rev. D 85, 094510 (2012).

[115] J. W. Chen, S. D. Cohen, X. Ji, H. W. Lin, and J. H. Zhang, Nucl. Phys. B911, 246 (2016).

[116] C. Alexandrou, K. Cichy, M. Constantinou, K. Hadjiyiannakou, K. Jansen, F. Steffens, and C. Wiese, Phys. Rev. D 96, 014513 (2017).

[117] B. Yoon, M. Engelhardt, R. Gupta, T. Bhattacharya, J. R. Green, B. U. Musch, J. W. Negele, A. V. Pochinsky, A. Schäfer, and S. N. Syritsyn, Phys. Rev. D 96, 094508 (2017).

[118] K. Orginos, A. Radyushkin, J. Karpie, and S. Zafeiropoulos, Phys. Rev. D 96, 094503 (2017).
[119] B. Joó, J. Karpie, K. Orginos, A. Radyushkin, D. Richards, and S. Zafeiropoulos, J. High Energy Phys. 12 (2019) 081.

[120] U. D'Alesio, E. Leader, and F. Murgia, Phys. Rev. D 81, 036010 (2010).

[121] C. Lorcé and B. Pasquini, Phys. Rev. D 84, 034039 (2011).

[122] C. Lorcé and B. Pasquini, Phys. Lett. B 710, 486 (2012).

[123] J. C. Collins, Phys. Lett. B 536, 43 (2002).

[124] S. J. Brodsky, D. S. Hwang, and I. Schmidt, Nucl. Phys. B642, 344 (2002).

[125] S. J. Brodsky, D. S. Hwang, and I. Schmidt, Phys. Lett. B 530, 99 (2002).

[126] A. V. Belitsky, X. Ji, and F. Yuan, Nucl. Phys. B656, 165 (2003).

[127] C. J. Bomhof, P. J. Mulders, and F. Pijlman, Eur. Phys. J. C 47, 147 (2006).

[128] K. Goeke, A. Metz, and M. Schlegel, Phys. Lett. B 618, 90 (2005).

[129] A. Metz, P. Schweitzer, and T. Teckentrup, Phys. Lett. B 680, 141 (2009).

[130] P. V. Pobylitsa, arXiv:hep-ph/0301236.

[131] K. Goeke, A. Metz, P. V. Pobylitsa, and M. V. Polyakov, Phys. Lett. B 567, 27 (2003).

[132] G. Karl and J. E. Paton, Phys. Rev. D 30, 238 (1984).

[133] S. Wandzura and F. Wilczek, Phys. Lett. 72B, 195 (1977).

[134] J. Balla, M. V. Polyakov, and C. Weiss, Nucl. Phys. B510, 327 (1998).

[135] B. Dressler and M. V. Polyakov, Phys. Rev. D 61, 097501 (2000).

[136] D. V. Kiptily and M. V. Polyakov, Eur. Phys. J. C 37, 105 (2004).

[137] M. Göckeler, R. Horsley, W. Kurzinger, H. Oelrich, D. Pleiter, P. E. L. Rakow, A. Schäfer, and G. Schierholz, Phys. Rev. D 63, 074506 (2001).

[138] M. Göckeler, R. Horsley, D. Pleiter, P. E. L. Rakow, A. Schäfer, G. Schierholz, H. Stuben, and J. M. Zanotti, Phys. Rev. D 72, 054507 (2005).

[139] K. Abe et al. (E143 Collaboration), Phys. Rev. D 58, 112003 (1998).

[140] P. L. Anthony et al. (E155 Collaboration), Phys. Lett. B 553, 18 (2003).

[141] A. Airapetian et al. (HERMES Collaboration), Eur. Phys. J. C 72, 1921 (2012).

[142] P. Ball and V. M. Braun, Phys. Rev. D 54, 2182 (1996).

[143] J. Blümlein and N. Kochelev, Phys. Lett. B 381, 296 (1996).

[144] J. Blümlein and N. Kochelev, Nucl. Phys. B498, 285 (1997).

[145] J. Soffer and O. V. Teryaev, Phys. Rev. D 56, R1353 (1997).

[146] J. Blümlein and A. Tkabladze, Nucl. Phys. B553, 427 (1999).

[147] N. Kivel, M. V. Polyakov, A. Schäfer, and O. V. Teryaev, Phys. Lett. B 497, 73 (2001).

[148] A. V. Belitsky and D. Müller, Nucl. Phys. B589, 611 (2000).

[149] I. V. Anikin and O. V. Teryaev, Phys. Lett. B 509, 95 (2001). 
[150] A. V. Radyushkin and C. Weiss, Phys. Rev. D 64, 097504 (2001).

[151] A. V. Efremov, K. Goeke, and P. Schweitzer, Phys. Lett. B 522, 37 (2001).

[152] A. V. Efremov, K. Goeke, and P. Schweitzer, Eur. Phys. J. C 24, 407 (2002).

[153] A. Kotzinian, B. Parsamyan, and A. Prokudin, Phys. Rev. D 73, 114017 (2006).

[154] H. Avakian, A. V. Efremov, K. Goeke, A. Metz, P. Schweitzer, and T. Teckentrup, Phys. Rev. D 77, 014023 (2008).

[155] A. Accardi, A. Bacchetta, W. Melnitchouk, and M. Schlegel, J. High Energy Phys. 11 (2009) 093.

[156] S. Bastami, H. Avakian, A. V. Efremov, A. Kotzinian, B. U. Musch, B. Parsamyan, A. Prokudin, M. Schlegel, G. Schnell, P. Schweitzer, and K. Tezgin, J. High Energy Phys. 06 (2019) 007.

[157] S. Bastami, L. Gamberg, B. Parsamyan, B. Pasquini, A. Prokudin, and P. Schweitzer, arXiv:2005.14322.

[158] Y. Koike, K. Tanaka, and S. Yoshida, Phys. Lett. B 668 , 286 (2008).

[159] S. J. Brodsky, P. Hoyer, N. Marchal, S. Peigne, and F. Sannino, Phys. Rev. D 65, 114025 (2002).

[160] M. Glück, E. Reya, and A. Vogt, Eur. Phys. J. C 5, 461 (1998).

[161] A. D. Martin, R. G. Roberts, W. J. Stirling, and R. S. Thorne, Eur. Phys. J. C 4, 463 (1998).

[162] A. D. Martin, W. J. Stirling, R. S. Thorne, and G. Watt, Eur. Phys. J. C 63, 189 (2009).

[163] M. Glück, E. Reya, M. Stratmann, and W. Vogelsang, Phys. Rev. D 63, 094005 (2001).

[164] E. Leader, A. V. Sidorov, and D. B. Stamenov, Phys. Rev. D 73, 034023 (2006).

[165] L. A. Harland-Lang, A. D. Martin, P. Motylinski, and R. S. Thorne, Eur. Phys. J. C 75, 204 (2015).

[166] S. Alekhin, J. Blümlein, S. Moch, and R. Placakyte, Phys. Rev. D 96, 014011 (2017).

[167] R. D. Ball et al. (NNPDF Collaboration), Eur. Phys. J. C 77, 663 (2017).

[168] N. Sato, C. Andres, J. J. Ethier, and W. Melnitchouk (JAM Collaboration), Phys. Rev. D 101, 074020 (2020).
[169] T. J. Hou, J. Gao, T. J. Hobbs, K. Xie, S. Dulat, M. Guzzi, J. Huston, P. Nadolsky, J. Pumplin, C. Schmidt, I. Sitiwaldi, D. Stump, and C. P. Yuan, Phys. Rev. D 103, 014013 (2021)..

[170] D. de Florian, R. Sassot, M. Stratmann, and W. Vogelsang, Phys. Rev. Lett. 113, 012001 (2014).

[171] E. R. Nocera, R. D. Ball, S. Forte, G. Ridolfi, and J. Rojo (NNPDF Collaboration), Nucl. Phys. B887, 276 (2014).

[172] E. Leader, A. V. Sidorov, and D. B. Stamenov, Phys. Rev. D 91, 054017 (2015).

[173] J. J. Ethier, N. Sato, and W. Melnitchouk, Phys. Rev. Lett. 119, 132001 (2017).

[174] D. De Florian, G. A. Lucero, R. Sassot, M. Stratmann, and W. Vogelsang, Phys. Rev. D 100, 114027 (2019).

[175] J. J. Ethier and E. R. Nocera, Annu. Rev. Nucl. Part. Sci. 70, 43 (2020).

[176] E. C. Aschenauer, I. Borsa, G. Lucero, A. S. Nunes, and R. Sassot, Phys. Rev. D 102, 094018 (2020).

[177] L. P. Gamberg, D. S. Hwang, A. Metz, and M. Schlegel, Phys. Lett. B 639, 508 (2006).

[178] Y. V. Kovchegov and M. D. Sievert, Phys. Rev. D 99, 054032 (2019).

[179] Y. V. Kovchegov and M. D. Sievert, Phys. Rev. D 99, 054033 (2019).

[180] A. V. Efremov, K. Goeke, and P. Schweitzer, Phys. Rev. D 67, 114014 (2003).

[181] A. Courtoy, arXiv:1405.7659.

[182] M. Burkardt and Y. Koike, Nucl. Phys. B632, 311 (2002).

[183] A. V. Efremov and P. Schweitzer, J. High Energy Phys. 08 (2003) 006.

[184] F. Aslan and M. Burkardt, Phys. Rev. D 101, 016010 (2020).

[185] J. P. Ma and G. P. Zhang, Phys. Lett. B 811, 135947 (2020).

[186] S. Bhattacharya, K. Cichy, M. Constantinou, A. Metz, A. Scapellato, and F. Steffens, Phys. Rev. D 102, 114025 (2020).

[187] M. Burkardt, Phys. Rev. D 88, 114502 (2013).

[188] V. Barone, A. Drago, and P. G. Ratcliffe, Phys. Rep. 359, 1 (2002).

[189] G. A. Miller, Phys. Rev. C 76, 065209 (2007). 